\title{
Cyclic Codes and Sequences: the Generalized Kasami Case
}

\author{
October 30, 2018
}

Jinquan Luo Yuansheng Tang and Hongyu Wang

\footnotetext{
${ }^{0}$ The authors are with the School of Mathematics, Yangzhou University, Jiangsu Province, 225009, China

J.Luo is also with the Division of Mathematics, School of Physics and Mathematical Sciences, Nanyang Technological University, Singapore.

E-mail addresses: jqluo@ntu.edu.sg,ystang@yzu.edu.cn, hywang@yzu.edu.cn.
} 


\section{Abstract}

Let $q=2^{n}$ with $n=2 m$. Let $1 \leq k \leq n-1$ and $k \neq m$. In this paper we determine the value distribution of following exponential sums

$$
\sum_{x \in \mathbb{F}_{q}}(-1)^{\operatorname{Tr}_{1}^{m}\left(\alpha x^{2^{m}+1}\right)+\operatorname{Tr}_{1}^{n}\left(\beta x^{2^{k}+1}\right)} \quad\left(\alpha \in \mathbb{F}_{2^{m}}, \beta \in \mathbb{F}_{q}\right)
$$

and

$$
\sum_{x \in \mathbb{F}_{q}}(-1)^{\operatorname{Tr}_{1}^{m}\left(\alpha x^{2^{m}+1}\right)+\operatorname{Tr}_{1}^{n}\left(\beta x^{2^{k}+1}+\gamma x\right)} \quad\left(\alpha \in \mathbb{F}_{2^{m}}, \beta, \gamma \in \mathbb{F}_{q}\right)
$$

where $\operatorname{Tr}_{1}^{n}: \mathbb{F}_{q} \rightarrow \mathbb{F}_{2}$ and $\operatorname{Tr}_{1}^{m}: \mathbb{F}_{p^{m}} \rightarrow \mathbb{F}_{2}$ are the canonical trace mappings. As applications:

(1). We determine the weight distribution of the binary cyclic codes $\mathcal{C}_{1}$ and $\mathcal{C}_{2}$ with parity-check polynomials $h_{2}(x) h_{3}(x)$ and $h_{1}(x) h_{2}(x) h_{3}(x)$ respectively where $h_{1}(x), h_{2}(x)$ and $h_{3}(x)$ are the minimal polynomials of $\pi^{-1}, \pi^{-\left(2^{k}+1\right)}$ and $\pi^{-\left(2^{m}+1\right)}$ over $\mathbb{F}_{2}$ respectively for a primitive element $\pi$ of $\mathbb{F}_{q}$.

(2). We determine the correlation distribution among a family of m-sequences.

This paper is the binary version of Luo, Tang and Wang[16] and extends the results in Kasami[17], Van der Vlugt [27] and Zeng, Liu and $\mathrm{Hu}$ [31.

Index terms: Exponential sum, Cyclic code, Quadratic form, Weight distribution, Correlation distribution 


\section{Introduction}

Basic results on finite fields could be found in [20]. The following standard notations are fixed throughout this paper except for specific statements.

- Let $n$ be an even integer, $m=n / 2$ and $q=2^{n}$.

- Let $\mathbb{F}_{2^{l}}$ be the finite field of order $2^{l}, \mathbb{F}_{2^{l}}^{*}$ be the set consisting of nonzero element and $\operatorname{Tr}_{i}^{j}: \mathbb{F}_{p^{i}} \rightarrow \mathbb{F}_{p^{j}}$ be the trace mapping for $i \mid j$.

- Let $k$ be a positive integer, $1 \leq k \leq n-1$ and $k \neq m$. Let $d=\operatorname{gcd}(m, k)$ and $d^{\prime}=\operatorname{gcd}(m+k, 2 k), q_{0}=2^{d}$, and $s=n / d$.

- Let $\pi$ be a primitive element of $\mathbb{F}_{q}^{*}$.

For binary cyclic code $\mathcal{C}$ with length $l$, let $A_{i}$ be the number of codewords in $\mathcal{C}$ with Hamming weight $i$. The weight distribution $\left\{A_{0}, A_{1}, \cdots, A_{l}\right\}$ is an important research object for both theoretical and application interests in coding theory. Classical coding theory reveals that the weight of each codeword can be expressed by binary exponential sums so that the weight distribution of $\mathcal{C}$ can be determined if the corresponding exponential sums can be calculated explicitly (Kasami [17], [18, [19], van der Vlugt [27], [31]).

In general, let $q=2^{n}, \mathcal{C}$ be the binary cyclic code with length $l=q-1$ and parity-check polynomial

$$
h(x)=h_{1}(x) \cdots h_{u}(x) \quad(u \geq 1)
$$

where $h_{i}(x)(1 \leq i \leq u)$ are distinct irreducible polynomials in $\mathbb{F}_{2}[x]$ with the same degree $e_{i}(1 \leq i \leq u)$, then $\operatorname{dim}_{\mathbb{F}_{2}} \mathcal{C}=\sum_{i=1}^{u} e_{i}$.Let $\pi^{-s_{i}}$ be a zero of $h_{i}(x)$, $1 \leq s_{i} \leq q-2(1 \leq i \leq u)$. Then the codewords in $\mathcal{C}$ can be expressed by

$$
c\left(\alpha_{1}, \cdots, \alpha_{u}\right)=\left(c_{0}, c_{1}, \cdots, c_{l-1}\right) \quad\left(\alpha_{1}, \cdots, \alpha_{u} \in \mathbb{F}_{q}\right)
$$


where $c_{i}=\sum_{\lambda=1}^{u} \operatorname{Tr}_{1}^{n}\left(\alpha_{\lambda} \pi^{i s_{\lambda}}\right)(0 \leq i \leq l-1)$. Therefore the Hamming weight of the codeword $c=c\left(\alpha_{1}, \cdots, \alpha_{u}\right)$ is

$$
\begin{aligned}
w_{H}(c) & =\#\left\{i \mid 0 \leq i \leq l-1, c_{i} \neq 0\right\} \\
& =l-\#\left\{i \mid 0 \leq i \leq l-1, c_{i}=0\right\} \\
& =l-\frac{1}{2} \sum_{i=0}^{l-1} \sum_{a=0}^{1}(-1)^{a \cdot \operatorname{Tr}_{1}^{n}\left(\sum_{\lambda=1}^{u} \alpha_{\lambda} \pi^{i s_{\lambda}}\right)} \\
& =l-\frac{l}{2}-\frac{1}{2} \sum_{x \in \mathbb{F}_{q}^{*}}(-1)^{\operatorname{Tr}_{1}^{n}(f(x))} \\
& =\frac{l}{2}+\frac{1}{2}-\frac{1}{2} S\left(\alpha_{1}, \cdots, \alpha_{u}\right) \\
& =2^{n-1}-\frac{1}{2} S\left(\alpha_{1}, \cdots, \alpha_{u}\right)
\end{aligned}
$$

where $f(x)=\alpha_{1} x^{s_{1}}+\alpha_{2} x^{s_{2}}+\cdots+\alpha_{u} x^{s_{u}} \in \mathbb{F}_{q}[x], \mathbb{F}_{q}^{*}=\mathbb{F}_{q} \backslash\{0\}$, and

$$
S\left(\alpha_{1}, \cdots, \alpha_{u}\right)=\sum_{x \in \mathbb{F}_{q}}(-1)^{\operatorname{Tr}_{1}^{n}\left(\alpha_{1} x^{s_{1}}+\cdots+\alpha_{u} x^{s_{u}}\right)} .
$$

In this way, the weight distribution of cyclic code $\mathcal{C}$ can be derived from the explicit evaluating of the exponential sums

$$
S\left(\alpha_{1}, \cdots, \alpha_{u}\right) \quad\left(\alpha_{1}, \cdots, \alpha_{u} \in \mathbb{F}_{q}\right) \text {. }
$$

Let $h_{1}(x), h_{2}(x)$ and $h_{3}(x)$ be the minimal polynomials of $\pi^{-1}, \pi^{-\left(2^{k}+1\right)}$ and $\pi^{-\left(2^{m}+1\right)}$ over $\mathbb{F}_{2}$ respectively. Then

$$
\operatorname{deg} h_{i}(x)=n \text { for } i=1,2 \text { and } \operatorname{deg} h_{3}(x)=m
$$

Let $\mathcal{C}_{1}$ and $\mathcal{C}_{2}$ be the binary cyclic codes with length $l=q-1$ and parity-check polynomials $h_{2}(x) h_{3}(x)$ and $h_{1}(x) h_{2}(x) h_{3}(x)$ respectively. It is a consequence that $\mathcal{C}_{1}\left(\mathcal{C}_{2}\right.$, resp.) is the dual of the binary $\mathrm{BCH}$ code with designed distance 5 (7, resp.) whose zeroes include $\pi^{2^{m}+1}$ and $\pi^{2^{k}+1}\left(\pi^{2^{m}+1}, \pi^{2^{k}+1}\right.$ and $\pi$, resp.). From (2), we know that the dimensions of $\mathcal{C}_{1}$ and $\mathcal{C}_{2}$ over $\mathbb{F}_{p^{t}}$ are $3 n / 2$ and $5 n / 2$ respectively. 
For $\alpha \in \mathbb{F}_{2^{m}},(\beta, \gamma) \in \mathbb{F}_{q}^{2}$, define the exponential sums

$$
T(\alpha, \beta)=\sum_{x \in \mathbb{F}_{q}}(-1)^{\operatorname{Tr}_{1}^{m}\left(\alpha x^{2^{m}+1}\right)+\operatorname{Tr}_{1}^{n}\left(\beta x^{2^{k}+1}\right)}
$$

and

$$
S(\alpha, \beta, \gamma)=\sum_{x \in \mathbb{F}_{q}}(-1)^{\operatorname{Tr}_{1}^{m}\left(\alpha x^{2^{m}+1}\right)+\operatorname{Tr}_{1}^{n}\left(\beta x^{2^{k}+1}+\gamma x\right)} .
$$

Then the complete weight distributions of $\mathcal{C}_{1}$ and $\mathcal{C}_{2}$ can be derived from the explicit evaluation of $T(\alpha, \beta)$ and $S(\alpha, \beta, \gamma)$.

Another application of binary exponential sums is to obtain the cross correlation and auto-correlation distribution among binary sequences. Let $\mathcal{F}$ be a collection of binary m-sequences of period $q-1$ defined by

$$
\mathcal{F}=\left\{\left\{a_{i}(t)\right\}_{i=0}^{q-2} \mid 0 \leq i \leq L-1\right\}
$$

The correlation function of $a_{i}$ and $a_{j}$ for a shift $\tau$ is defined by

$$
M_{i, j}(\tau)=\sum_{\lambda=0}^{q-2}(-1)^{a_{i}(\lambda)-a_{j}(\lambda+\tau)} \quad(0 \leq \tau \leq q-2) .
$$

Binary sequences with low cross correlation and auto-correlation are widely used in Code Division Multiple Access(CDMA) spread spectrum(see Paterson 24, Simon, Omura and Scholtz[26]). Pairs of binary m-sequences with few-valued auto and cross correlations have been extensively studied for several decades, see Canteaut, Charpin and Dobbertin [1], Cusick and Dobbertin [4], Ding, Helleseth and Lam[5], Ding, Helleseth and Martinsen[6], Dobbertin, Felke, Helleseth and Rosendahl [7], Gold [8], Helleseth [10, [11, Helleseth, Kholosha and Ness 12], Helleseth and Kumar [13], Hollmann and Xiang [14], Ness and Helleseth[23], Niho[24], Rosendahl[25], Yu and Gong[28]-[29] and references therein.

Define the collection of sequences

$$
\mathcal{F}_{1}=\left\{a_{\alpha, \beta}=\left(a_{\alpha, \beta}\left(\pi^{\lambda}\right)\right)_{\lambda=0}^{q-2} \mid \alpha \in \mathbb{F}_{2^{m}}, \beta \in \mathbb{F}_{q}\right\}
$$

where $a_{\alpha, \beta}\left(\pi^{\lambda}\right)=\operatorname{Tr}_{1}^{m}\left(\alpha \pi^{\lambda\left(2^{m}+1\right)}\right)+\operatorname{Tr}_{1}^{n}\left(\beta \pi^{\lambda\left(2^{k}+1\right)}+\pi^{\lambda}\right)$.

If $m / d$ or $k / d$ is even, define

$$
\mathcal{F}_{2}=\left\{a_{\beta}=\left(a_{\beta}\left(\pi^{\lambda}\right)\right)_{\lambda=0}^{q-2} \mid \beta=\pi^{i} \text { for } 0 \leq i \leq 2^{m}-2\right\}
$$


where $a_{\beta}\left(\pi^{\lambda}\right)=\operatorname{Tr}_{1}^{m}\left(\pi^{\lambda\left(2^{m}+1\right)}\right)+\operatorname{Tr}_{1}^{n}\left(\beta \pi^{\lambda\left(2^{k}+1\right)}\right)$.

If $k / d$ is even, define $\mathcal{F}_{3}=\{a\}$ with $a=\left(\operatorname{Tr}_{1}^{n}\left(\pi^{\lambda\left(2^{k}+1\right)}\right)\right)_{\lambda=0}^{q-2}$.

In this correspondence we will study the following collection of m-sequences with period $q-1$

$$
\mathcal{F}= \begin{cases}\mathcal{F}_{1}, & \text { if } m / d \text { and } k / d \text { are both odd } \\ \mathcal{F}_{1} \cup \mathcal{F}_{2}, & \text { if } m / d \text { is even } \\ \mathcal{F}_{1} \cup \mathcal{F}_{2} \cup \mathcal{F}_{3}, & \text { if } k / d \text { is even. }\end{cases}
$$

It is easy to verify that the sequences in $\mathcal{F}$ are all cyclic inequivalent and maximal with size $q-1$. Several particular cases of the cyclic code $\mathcal{C}_{2}$ or the related sequences collection $\mathcal{F}$ have been investigated, for instance:

- The binary code $\mathcal{C}_{2}$ with $k=m \pm 1$ is nothing but the classical Kasami code, see Kasami [17].

- As for the binary code $\mathcal{C}_{2}$ with $k=1$, its minimal distance is obtained by Lahtonen [21, Moreno and Kumar [22]. Its weight distribution is determined eventually in van der Vlugt [27].

- For the case $(k, n)=2$ if $m$ is odd, or $(k, n)=1$ if $m$ is even, the binary code $\mathcal{C}_{2}$ and the related family of generalized Kasami sequences have been studied, see Zeng, Liu and $\mathrm{Hu}$ [31].

- In the case $p$ odd prime and $\operatorname{gcd}(m, k)=\operatorname{gcd}(m+k, 2 k)=d$ being odd, the weight distribution of $\mathcal{C}_{2}$ and correlation distribution of corresponding sequences have been fully determined, see Zeng, $\mathrm{Li}$ and $\mathrm{Hu}$ [30].

This paper is presented as follows. In Section 2 we introduce some preliminaries and give auxiliary results. In Section 3 we will give the value distribution of $T(\alpha, \beta)$ for $\alpha \in \mathbb{F}_{2^{m}}, \beta \in \mathbb{F}_{q}$ and the weight distribution of $\mathcal{C}_{1}$. In Section 3 we will determine the value distribution of $S(\alpha, \beta, \gamma)$, the correlation distribution among the sequences in $\mathcal{F}$, and then the weight distribution of $\mathcal{C}_{2}$. Most proofs of lemmas and theorems are presented in several appendices. The main tools are quadratic form theory over finite fields of characteristic 2, some moment identities on $T(\alpha, \beta)$ and a class of Artin-Schreier curves. 


\section{Preliminaries}

We follow the notations in Section 1. The first machinery to determine the values of exponential sums $T(\alpha, \beta)\left(\alpha \in \mathbb{F}_{p^{m}}, \beta \in \mathbb{F}_{q}\right)$ defined in (3) is quadratic form theory over $\mathbb{F}_{q_{0}}$.

Let $H$ be an $s \times s$ matrix over $\mathbb{F}_{q_{0}}$. For the quadratic form

$$
F: \mathbb{F}_{q_{0}}^{s} \rightarrow \mathbb{F}_{q_{0}}, \quad F(x)=X H X^{T} \quad\left(X=\left(x_{1}, \cdots, x_{s}\right) \in \mathbb{F}_{q_{0}}^{s}\right),
$$

define $r_{F}$ of $F$ to be the rank of the skew-symmetric matrix $H+H^{T}$. Then $r_{F}$ is even.

Lemma 1. For the quadratic form $F(X)=X H X^{T}$ defined in ([6),

$$
\sum_{X \in \mathbb{F}_{q_{0}}^{s}} \zeta_{p}^{\operatorname{Tr}_{1}^{d}(F(X))}= \pm q_{0}^{s-\frac{r_{F}}{2}} \text { or } 0
$$

Moreover, if $r_{F}=s$, then

$$
\sum_{X \in \mathbb{F}_{q_{0}}^{s}} \zeta_{p}^{\operatorname{Tr}_{1}^{d}(F(X))}= \pm q_{0}^{\frac{s}{2}}
$$

Proof. We can calculate

$$
\begin{gathered}
\left(\sum_{X \in \mathbb{F}_{q_{0}}^{s}}(-1)^{\operatorname{Tr}_{1}^{d}(F(X))}\right)^{2}=\sum_{X, Z \in \mathbb{F}_{q_{0}}^{s}}(-1)^{\operatorname{Tr}_{1}^{d}\left(X H X^{T}+(X+Z) H(X+Z)^{T}\right)} \\
=\sum_{Z \in \mathbb{F}_{q_{0}}^{s}}(-1)^{\operatorname{Tr}_{1}^{d}\left(Z H Z^{T}\right)} \sum_{X \in \mathbb{F}_{q_{0}}^{s}}(-1)^{\operatorname{Tr}_{1}^{d}\left(Z\left(H+H^{T}\right) X^{T}\right)}
\end{gathered}
$$

The inner sum is zero unless $Z\left(H+H^{T}\right)=0$. Define

$$
\mathcal{Z}=\left\{Z \in \mathbb{F}_{q_{0}}^{s} \mid Z\left(H+H^{T}\right)=0\right\} .
$$

Then the map

$$
\begin{aligned}
\eta: \mathcal{Z} & \longrightarrow \mathbb{F}_{2} \\
Z & \mapsto \operatorname{Tr}_{1}^{d}\left(Z H Z^{T}\right)
\end{aligned}
$$

is an additive group homomorphism. 
If $\eta$ is surjective, then there are exactly one half $z \in \mathcal{Z}$ mapping to 0 and 1 respectively. Hence $\sum_{X \in \mathbb{F}_{q_{0}}^{s}}(-1)^{\operatorname{Tr}_{1}^{d}(F(X))}=0$. Otherwise $\operatorname{Im}(\eta)=\{0\}$ and $\left(\sum_{X \in \mathbb{F}_{q_{0}}^{s}}(-1)^{\operatorname{Tr}_{1}^{d}(F(X))}\right)^{2}=q_{0}^{s} \cdot q_{0}^{s-r_{F}}$. Hence $\sum_{X \in \mathbb{F}_{q_{0}}^{s}}(-1)^{\operatorname{Tr}_{1}^{d}(F(X))}= \pm q_{0}^{s-\frac{r_{F}}{2}}$.

If $r_{F}=s$, then $\mathcal{Z}=\{0\}$ and $\operatorname{Im}(\eta)=\{0\}$. Therefore $\sum_{X \in \mathbb{F}_{q_{0}}^{s}}(-1)^{\operatorname{Tr}_{1}^{d}(F(X))}=$ $\pm q_{0}^{\frac{s}{2}}$

The following result, which has been proven in [20, Chap. 6, will be used in Section 4 .

Lemma 2. For the fixed quadratic form defined in (6), the value distribution of $\sum_{X \in \mathbb{F}_{q_{0}}^{s}}(-1)^{\operatorname{Tr}_{1}^{d}\left(F(X)+A X^{T}\right)}$ when $A$ runs through $\mathbb{F}_{q_{0}}^{s}$ is shown as following

$$
\begin{array}{cc}
\text { value } & \text { multiplicity } \\
0 & q_{0}^{s}-q_{0}^{r_{F}} \\
q_{0}^{s-\frac{r_{F}}{2}} & \frac{1}{2}\left(q_{0}^{r_{F}}+q_{0}^{\frac{r_{F}}{2}}\right) \\
-q_{0}^{s-\frac{r_{F}}{2}} & \frac{1}{2}\left(q_{0}^{r_{F}}-q_{0}^{\frac{r_{F}}{2}}\right)
\end{array}
$$

Since $s=n / d$, the field $\mathbb{F}_{q}$ is a vector space over $\mathbb{F}_{q_{0}}$ with dimension $s$. We fix a basis $v_{1}, \cdots, v_{s}$ of $\mathbb{F}_{q}$ over $\mathbb{F}_{q_{0}}$. Then each $x \in \mathbb{F}_{q}$ can be uniquely expressed as

$$
x=x_{1} v_{1}+\cdots+x_{s} v_{s} \quad\left(x_{i} \in \mathbb{F}_{q_{0}}\right) .
$$

Thus we have the following $\mathbb{F}_{q_{0}}$-linear isomorphism:

$$
\mathbb{F}_{q} \stackrel{\sim}{\rightarrow} \mathbb{F}_{q_{0}}^{s}, \quad x=x_{1} v_{1}+\cdots+x_{s} v_{s} \mapsto X=\left(x_{1}, \cdots, x_{s}\right) .
$$

With this isomorphism, a function $f: \mathbb{F}_{q} \rightarrow \mathbb{F}_{q_{0}}$ induces a function $F: \mathbb{F}_{q_{0}}^{s} \rightarrow \mathbb{F}_{q_{0}}$ where for $X=\left(x_{1}, \cdots, x_{s}\right) \in \mathbb{F}_{q_{0}}^{s}, F(X)=f(x)$ with $x=x_{1} v_{1}+\cdots+x_{s} v_{s}$. In this way, function $f(x)=\operatorname{Tr}_{d}^{n}(\gamma x)$ for $\gamma \in \mathbb{F}_{q}$ induces a linear form

$$
F(X)=\operatorname{Tr}_{d}^{n}(\gamma x)=\sum_{i=1}^{s} \operatorname{Tr}_{d}^{n}\left(\gamma v_{i}\right) x_{i}=A_{\gamma} X^{T}
$$


where $A_{\gamma}=\left(\operatorname{Tr}_{d}^{n}\left(\gamma v_{1}\right), \cdots, \operatorname{Tr}_{d}^{n}\left(\gamma v_{s}\right)\right)$, and $f_{\alpha, \beta}(x)=\operatorname{Tr}_{d}^{m}\left(\alpha x^{p^{m}+1}\right)+\operatorname{Tr}_{d}^{n}\left(\beta x^{p^{k}+1}\right)$ for $\alpha \in \mathbb{F}_{p^{m}}, \beta \in \mathbb{F}_{q}$ induces a quadratic form

$$
F_{\alpha, \beta}(X)=X H_{\alpha, \beta} X^{T}
$$

From Lemma 1, for $\alpha \in \mathbb{F}_{2^{m}},(\beta, \gamma) \in \mathbb{F}_{q}^{2}$, in order to determine the values of

$$
T(\alpha, \beta)=\sum_{x \in \mathbb{F}_{q}}(-1)^{\operatorname{Tr}_{1}^{m}\left(\alpha x^{2^{m}+1}\right)+\operatorname{Tr}_{1}^{n}\left(\beta x^{2^{k}+1}\right)}=\sum_{X \in \mathbb{F}_{q_{0}}^{s}}(-1)^{\operatorname{Tr}_{1}^{d}\left(X H_{\alpha, \beta} X^{T}\right)}
$$

and

$$
S(\alpha, \beta, \gamma)=\sum_{x \in \mathbb{F}_{q}}(-1)^{\operatorname{Tr}_{1}^{m}\left(\alpha x^{2^{m}+1}\right)+\operatorname{Tr}_{1}^{n}\left(\beta x^{2^{k}+1}+\gamma x\right)}=\sum_{X \in \mathbb{F}_{q_{0}}^{s}}(-1)^{\operatorname{Tr}_{1}^{d}\left(X H_{\alpha, \beta} X^{T}+A_{\gamma} X^{T}\right)},
$$

we need to determine the rank of $H_{\alpha, \beta}+H_{\alpha, \beta}^{T}$ over $\mathbb{F}_{q_{0}}$.

Define $d^{\prime}=\operatorname{gcd}(m+k, 2 k)$. Then an easy observation shows

$$
d^{\prime}= \begin{cases}2 d, & \text { if } m / d \text { and } k / d \text { are both odd; } \\ d, & \text { otherwise }\end{cases}
$$

Special case of the subsequent result has been proven in [31].

Lemma 3. For $(\alpha, \beta) \in \mathbb{F}_{p^{m}} \times \mathbb{F}_{q} \backslash\{(0,0)\}$, let $r_{\alpha, \beta}$ be the rank of $H_{\alpha, \beta}+H_{\alpha, \beta}^{T}$. Then we have

(i). if $d^{\prime}=d$, then the possible values of $r_{\alpha, \beta}$ are $s, s-2$.

(ii). if $d^{\prime}=2 d$, then the possible values of $r_{\alpha, \beta}$ are $s, s-2, s-4$.

Moreover, let $n_{i}$ be the number of $(\alpha, \beta)$ with $r_{\alpha, \beta}=s-i$. In the case $d^{\prime}=d$, we have

$$
\begin{aligned}
& n_{0}=\left(2^{n+2 d}-2^{n+d}-2^{n}+2^{m+2 d}-2^{m+d}+2^{2 d}\right)\left(2^{m}-1\right) /\left(2^{2 d}-1\right) \\
& n_{2}=\left(2^{m+d}-1\right)\left(2^{n}-1\right) /\left(2^{2 d}-1\right) .
\end{aligned}
$$

We need to introduce some results to prove Lemma 3 .

Lemma 4. (see Bluher [1], Theorem 5.4 and 5.6) Let $g(z)=z^{p^{h}+1}-b z+b$ with $b \in \mathbb{F}_{p^{l}}^{*}$ and $e=\operatorname{gcd}(h, l)$. Then we have 
(i). the number of the solutions to $g(z)=0$ in $\mathbb{F}_{p^{l}}$ is $0,1,2$ or $p^{e}+1$.

(ii). if $z_{0}$ is the unique solution in $\mathbb{F}_{p^{l}}^{*}$, then $\left(z_{0}-1\right)^{\frac{p^{l}-1}{p^{e}-1}}=1$.

(iii). denote by $N_{i}$ the number of $b \in \mathbb{F}_{p^{l}}^{*}$ such that $g(z)=0$ has exactly $i$ roots in $\mathbb{F}_{2^{l}}$. Then we get

- if $l / e$ is even, then

$$
N_{0}=\frac{2^{l+e}-2^{e}}{2\left(2^{e}+1\right)}, \quad N_{1}=2^{l-e}, \quad N_{2}=\frac{\left(2^{e}-2\right)\left(2^{l}-1\right)}{2\left(2^{e}-1\right)}, \quad N_{2^{e}+1}=\frac{2^{l-e}-2^{e}}{2^{2 e}-1} .
$$

- if $l / e$ is odd, then

$$
N_{0}=\frac{2^{l+e}+2^{e}}{2\left(2^{e}+1\right)}, \quad N_{1}=2^{l-e}-1, \quad N_{2}=\frac{\left(2^{e}-2\right)\left(2^{l}-1\right)}{2\left(2^{e}-1\right)}, \quad N_{2^{e}+1}=\frac{2^{l-e}-1}{2^{2 e}-1} .
$$

The following lemma has been proven in [1], 30] and [31]. We will repeat part of the proof for self-containing.

Lemma 5. Let $\psi_{\alpha, \beta}(z)=\beta^{2^{n-k}} z^{2^{m-k}+1}+\alpha z+\beta$ with $\alpha \in \mathbb{F}_{2^{m}}^{*}, \beta \in \mathbb{F}_{q}^{*}$. Then

(i). $\psi_{\alpha, \beta}(z)=0$ has either $0,1,2$ or $2^{d^{\prime}}+1$ solutions in $\mathbb{F}_{q}$.

(ii). If $z_{1}, z_{2}$ are two solutions of $\psi_{\alpha, \beta}(z)=0$ in $\mathbb{F}_{q}$, then $\left(z_{1} z_{2}\right)^{\frac{q-1}{2^{d}-1}}=1$.

(iii). If $\psi_{\alpha, \beta}(z)=0$ has $2^{d^{\prime}}+1$ solutions in $\mathbb{F}_{q}$, then for any two solutions $z_{1}$ and $z_{2}$, we have $\left(z_{1} / z_{2}\right)^{\frac{q-1}{2^{\prime}-1}}=1$.

(iv). If $\psi_{\alpha, \beta}(z)=0$ has exactly one solution in $\mathbb{F}_{q}$, say $z_{0}$, then $z_{0}^{\frac{q-1}{2^{d}-1}}=1$.

Proof. (i). By scaling $y=\frac{\alpha}{\beta} z$ and $b=\frac{\alpha^{2^{m-k}+1}}{\beta^{2^{m-k}\left(2^{m}+1\right)}}$, we can rewrite the equation $\psi_{\alpha, \beta}(z)=0$ as

$$
g(y)=y^{2^{m-k}+1}+b y+b=0 .
$$

Since $b \in \mathbb{F}_{2^{m}}^{*} \subseteq \mathbb{F}_{q}^{*}$ and $\operatorname{gcd}(m-k, n)=\operatorname{gcd}(m-k, 2 k)=d^{\prime}$, then the result follows from Lemma 4 (i).

(ii). See [30], Prop.1 (2). 
(iii). Denote by $y_{i}=\frac{\alpha}{\beta} z_{i}$ for $i=1,2$. Since $\operatorname{gcd}(n, m-k)=d^{\prime}$, from [1], Theorem 4.6 (iv) we get $\left(y_{1} / y_{2}\right)^{\frac{q-1}{2^{d^{\prime}}-1}}=1$ which is equivalent to $\left(z_{1} / z_{2}\right)^{\frac{q-1}{2^{d^{\prime}}-1}}=1$.

(iv). See [30], Prop.1 (3).

Remark. (i). If $\psi_{\alpha, \beta}(z)=0$ has exactly one or $2^{d^{\prime}}+1$ solutions in $\mathbb{F}_{q}$, then each solution is a $\left(2^{d}-1\right)$-th power in $\mathbb{F}_{q}$.

(ii). If $\psi_{\alpha, \beta}(z)=0$ has exactly two solutions in $\mathbb{F}_{q}$, then none or both of them are $\left(2^{d}-1\right)$-th powers in $\mathbb{F}_{q}$.

Proof of Lemma 3; (i). For $Y=\left(y_{1}, \cdots, y_{s}\right) \in \mathbb{F}_{q_{0}}^{s}, y=y_{1} v_{1}+\cdots+y_{s} v_{s} \in$ $\mathbb{F}_{q}$, we know that

$$
F_{\alpha, \beta}(X+Y)-F_{\alpha, \beta}(X)-F_{\alpha, \beta}(Y)=X\left(H_{\alpha, \beta}+H_{\alpha, \beta}^{T}\right) Y^{T}
$$

is equal to

$$
f_{\alpha, \beta}(x+y)-f_{\alpha, \beta}(x)-f_{\alpha, \beta}(y)=\operatorname{Tr}_{d}^{n}\left(y\left(\alpha x^{2^{m}}+\beta x^{2^{k}}+\beta^{2^{n-k}} x^{2^{n-k}}\right)\right) .
$$

Let

$$
\phi_{\alpha, \beta}(x)=\alpha x^{2^{m}}+\beta x^{2^{k}}+\beta^{2^{n-k}} x^{2^{n-k}} .
$$

Therefore,

$r_{\alpha, \beta}=r \Leftrightarrow$ the number of common solutions of $X\left(H_{\alpha, \beta}+H_{\alpha, \beta}^{T}\right) Y^{T}=0$ for all $Y \in \mathbb{F}_{q_{0}}^{s}$ is $q_{0}^{s-r}$, $\Leftrightarrow$ the number of common solutions of $\operatorname{Tr}_{d}^{n}\left(y \cdot \phi_{\alpha, \beta}(x)\right)=0$ for all $y \in \mathbb{F}_{q}$ is $q_{0}^{s-r}$, $\Leftrightarrow \phi_{\alpha, \beta}(x)=0$ has $q_{0}^{s-r}$ solutions in $\mathbb{F}_{q}$.

Since $\phi_{\alpha, \beta}(x)$ is a $2^{d}$-linearized polynomial, then the set of the zeroes to $\phi_{\alpha, \beta}(x)=0$ in $\mathbb{F}_{q}$, say $V$, forms an $\mathbb{F}_{2^{d} \text {-vector space. }}$

If $\alpha=0$ and $\beta \neq 0, \phi_{\alpha, \beta}(x)=0$ becomes $\beta x^{2^{k}}+\beta^{2^{n-k}} x^{2^{n-k}}=0$ and then $\beta^{2^{k}} x^{2^{2 k}}+\beta x=0$. In this case (14) has 1 or $2^{d^{\prime}}$ solutions according to $\beta^{1-2^{k}}$ is $\left(2^{2 d}-1\right)$-th power in $\mathbb{F}_{q}$ or not. Hence $r_{0, \beta}=s$ or $s-2$. More precisely, in the case $m / d$ is even, $\beta^{1-2^{k}}$ is $\left(2^{2 d}-1\right)$-th power in $\mathbb{F}_{q}^{*}$ if and only if $\beta$ is a $\left(p^{d}+1\right)$-th power in $\mathbb{F}_{q}^{*}$. Hence the numbers of $\beta \in \mathbb{F}_{q}^{*}$ such that $r_{0, \beta}=s-2$ and $s$ are exactly $\frac{2^{n}-1}{2^{d}+1}$ and $\frac{2^{d}\left(2^{n}-1\right)}{2^{d}+1}$ respectively. In the case $k / d$ is even, $\beta^{1-2^{k}}$ is always $\left(2^{2 d}-1\right)$-th power in $\mathbb{F}_{q}^{*}$ which follows that $r_{0, \beta}=s-2$ for $\beta \in \mathbb{F}_{q}^{*}$. If $\alpha \neq 0$ and $\beta=0$, then $\phi_{\alpha, 0}(x)=0$ has unique solution $x=0$ and as a consequence $r_{\alpha, 0}=s$. 
In the following we assume $\alpha \beta \neq 0$, we need to consider the nonzero solutions of $\phi_{\alpha, \beta}(x)=0$. By substituting $z=x^{2^{k}\left(p^{m-k}-1\right)}$ we get

$$
\psi_{\alpha, \beta}(z)=\beta^{2^{n-k}} z^{2^{m-k}+1}+\alpha z+\beta=0 .
$$

From Lemma 4, $\psi_{\alpha, \beta}(z)=0$ has either $0,1,2$ or $2^{d^{\prime}}+1$ solutions in $\mathbb{F}_{q}$. In the case $d^{\prime}=d$, by Lemma 5 and its Remark,

- if $\psi_{\alpha, \beta}(z)=0$ has no solution in $\mathbb{F}_{q}$, then $\phi_{\alpha, \beta}(x)=0$ has unique solution $x=0$ in $\mathbb{F}_{q}$ and $r_{\alpha, \beta}=s$.

- if $\psi_{\alpha, \beta}(z)=0$ has exactly one in $\mathbb{F}_{q}$ which is also a $\left(2^{d}-1\right)$-th power, then $r_{\alpha, \beta}=s-1$. But $s-1$ is not even that leads to a contradiction.

- if $\psi_{\alpha, \beta}(z)=0$ has two solutions in $\mathbb{F}_{q}$, then $\phi_{\alpha, \beta}(x)=0$ has one or $2\left(2^{d}-\right.$ $1)+1$ solutions in $\mathbb{F}_{q}$. Note that $2^{d+1}-1$ is not a $2^{d}$-th power which is impossible. Hence $r_{\alpha, \beta}=s$.

- if $\psi_{\alpha, \beta}(z)=0$ has $2^{d}+1$ solutions in $\mathbb{F}_{q}$, then they are all $\left(2^{d}-1\right)$-th power. Therefore $\phi_{\alpha, \beta}(x)=0$ has $\left(2^{d}+1\right)\left(2^{d}-1\right)+1=2^{2 d}$ solutions in $\mathbb{F}_{q}$ and $r_{\alpha, \beta}=s-2$.

Again by Lemma 4, $\psi_{\alpha, \beta}(z)=0$ has either $0,1,2$ or $2^{d}+1$ solutions in $\mathbb{F}_{2^{m}}$. Note that the solutions of $\psi_{\alpha, \beta}(z)=0$ have one-to-one correspondence with those of $g(y)=0$ by substituting $y=\frac{\alpha}{\beta} z$. If $y_{0} \in \mathbb{F}_{q} \backslash \mathbb{F}_{2^{m}}$ satisfying $g\left(y_{0}\right)=0$, then $g\left(y_{0}^{2^{m}}\right)=0$. Hence

- if $g(y)=0$ has no or two solutions in $\mathbb{F}_{2^{m}}$, then $g(y)=0$ and $\psi_{\alpha, \beta}(z)=0$ has no or two solutions in $\mathbb{F}_{q}$ and $r_{\alpha, \beta}=s$.

- $\psi_{\alpha, \beta}(z)=0$ has one or $2^{d}+1$ solutions in $\mathbb{F}_{2^{m}}$, then $g(y)=0$ and $\psi_{\alpha, \beta}(z)=0$ has $2^{d}+1$ solutions in $\mathbb{F}_{q}$ and $r_{\alpha, \beta}=s-2$.

For fixed $b$ and $\alpha \in \mathbb{F}_{2^{m}}^{*}$, the number of $\beta \in \mathbb{F}_{q}^{*}$ satisfying $b=\frac{\alpha^{2^{m-k}}+1}{\beta^{2^{m-k}\left(p^{m}+1\right)}}$ is $2^{m}+1$. Applying Lemma 4 with $l=m$ and $h=m+k$ we get that

- if $m / d$ is even, then

$$
\begin{aligned}
n_{0} & =\left(\frac{2^{m+d}-2^{d}}{2\left(2^{d}+1\right)}+\frac{\left(2^{d}-2\right)\left(2^{m}-1\right)}{2\left(2^{d}-1\right)}\right) \cdot\left(2^{m}-1\right) \cdot\left(2^{m}+1\right)+\frac{2^{d}\left(2^{n}-1\right)}{2^{d}+1}+\left(2^{m}-1\right) \\
& =\left(2^{n+2 d}-2^{n+d}-2^{n}+2^{m+2 d}-2^{m+d}+2^{2 d}\right)\left(2^{m}-1\right) /\left(2^{2 d}-1\right) .
\end{aligned}
$$


- if $k / d$ is even, then

$$
\begin{aligned}
n_{0} & =\left(\frac{2^{m+d}+2^{d}}{2\left(2^{d}+1\right)}+\frac{\left(2^{d}-2\right)\left(2^{m}-1\right)}{2\left(2^{d}-1\right)}\right) \cdot\left(2^{m}-1\right) \cdot\left(2^{m}+1\right)+\left(2^{m}-1\right) \\
& =\left(2^{n+2 d}-2^{n+d}-2^{n}+2^{m+2 d}-2^{m+d}+2^{2 d}\right)\left(2^{m}-1\right) /\left(2^{2 d}-1\right) .
\end{aligned}
$$

By $n_{0}+n_{2}=2^{3 m}-1$ we get the result.

In the case $d^{\prime}=2 d$, a similar argument gives the result.

In order to determine the multiplicity of each value of $T(\alpha, \beta)$ for $\alpha \in \mathbb{F}_{p^{m}}, \beta \in$ $\mathbb{F}_{q}$, we need the following result on moments of $T(\alpha, \beta)$.

Lemma 6. For the exponential sum $T(\alpha, \beta)$,

$$
\begin{aligned}
& \text { (i). } \sum_{\alpha \in \mathbb{F}_{2} m, \beta \in \mathbb{F}_{q}} T(\alpha, \beta)=2^{3 m} ; \\
& \text { (ii). } \sum_{\alpha \in \mathbb{F}_{2}, \beta \in \mathbb{F}_{q}} T(\alpha, \beta)^{2}= \begin{cases}2^{5 m} & \text { if } d^{\prime}=d \\
2^{3 m}\left(2^{n+d}+2^{n}-2^{d}\right) & \text { if } d^{\prime}=2 d ;\end{cases} \\
& \text { (iii). } \sum_{\alpha \in \mathbb{F}_{2} m, \beta \in \mathbb{F}_{q}} T(\alpha, \beta)^{3}= \begin{cases}2^{3 m}\left(2^{n+d}+2^{n}-2^{d}\right) & \text { if } d^{\prime}=d \\
2^{3 m}\left(2^{n+3 d}+2^{n}-2^{3 d}\right) & \text { if } d^{\prime}=2 d ;\end{cases}
\end{aligned}
$$

Proof. (i). We observe that

$$
\begin{aligned}
& \sum_{\alpha \in \mathbb{F}_{p}^{m}, \beta \in \mathbb{F}_{q}} T(\alpha, \beta)=\sum_{\substack{\alpha \in \mathbb{F}_{2}^{m}, \beta \in \mathbb{F}_{q} \\
\sum_{x \in \mathbb{F}_{q}}}}(-1)^{\operatorname{Tr}_{1}^{m}\left(\alpha x^{2^{m}+1}\right)+\operatorname{Tr}_{1}^{n}\left(\beta x^{2^{k}+1}\right)} \\
& \left.=\sum_{x \in \mathbb{F}_{q}} \sum_{\alpha \in \mathbb{F}_{2} m}(-1)^{\operatorname{Tr}_{1}^{m}\left(\alpha x^{2^{m}}+1\right.}\right) \sum_{\beta \in \mathbb{F}_{q}}(-1)^{\operatorname{Tr}_{1}^{n}\left(\beta x^{2^{k}+1}\right)}=q \cdot \sum_{\substack{\alpha \in \mathbb{F}_{2} m \\
x=0}}(-1)^{\operatorname{Tr}_{1}^{m}\left(\alpha x^{2^{m}+1}\right)}=2^{3 m} .
\end{aligned}
$$

(ii). We can calculate

$$
\begin{aligned}
\sum_{\alpha \in \mathbb{F}_{2} m, \beta \in \mathbb{F}_{q}} T(\alpha, \beta)^{2} & =\sum_{x, y \in \mathbb{F}_{q}} \sum_{\alpha \in \mathbb{F}_{2} m}(-1)^{\operatorname{Tr}_{1}^{m}\left(\alpha\left(x^{2^{m}+1}+y^{2^{m}+1}\right)\right)} \sum_{\beta \in \mathbb{F}_{q}}(-1)^{\operatorname{Tr}_{1}^{n}\left(\beta\left(x^{2^{k}+1}+y^{2^{k}+1}\right)\right)} \\
& =M_{2} \cdot 2^{3 m}
\end{aligned}
$$

where $M_{2}$ is the number of solutions to the equation

$$
\left\{\begin{array}{l}
x^{2^{m}+1}+y^{2^{m}+1}=0 \\
x^{2^{k}+1}+y^{2^{k}+1}=0
\end{array}\right.
$$

If $x y=0$ satisfying (16), then $x=y=0$. Otherwise $(x / y)^{2^{m}+1}=(x / y)^{2^{k}+1}=$ 1 which yields that $(x / y)^{2^{m-k}-1}=1$. Denote by $x=t y$. Since $\operatorname{gcd}(m-k, n)=d^{\prime}$, then $t \in \mathbb{F}_{2^{d^{\prime}}}^{*}$. 
- If $d^{\prime}=d$, then $t \in \mathbb{F}_{2^{d}}^{*}$ and (16) is equivalent to $x=y$. Hence $M_{2}=$ $1+(q-1)=q$.

- If $d^{\prime}=2 d$, then by (10) we get (16) is equivalent to $x^{2^{d}+1}=y^{2^{d}+1}$. Then we have $t^{2^{d}+1}=1$ which has $2^{d}+1$ solutions in $\mathbb{F}_{2^{d^{\prime}}}^{*}$. Therefore

$$
M_{2}=\left(2^{d}+1\right)\left(2^{n}-1\right)+1=2^{n+d}+2^{n}-2^{d} .
$$

(iii). We have

$$
\begin{aligned}
\sum_{\alpha \in \mathbb{F}_{2} m, \beta \in \mathbb{F}_{q}} T(\alpha, \beta)^{3} & =\sum_{x, y, z \in \mathbb{F}_{q}} \sum_{\alpha \in \mathbb{F}_{2} m}(-1)^{\operatorname{Tr}_{1}^{m}\left(\alpha\left(x^{2^{m}+1}+y^{2^{m}+1}+z^{2^{m}+1}\right)\right)} \sum_{\beta \in \mathbb{F}_{q}}(-1)^{\operatorname{Tr}_{1}^{n}\left(\beta\left(x^{2^{k}+1}+y^{2^{k}+1}+z^{2^{k}+1}\right)\right)} \\
& =M_{3} \cdot 2^{3 m}
\end{aligned}
$$

where $M_{3}$ is the number of solutions to the equation

$$
\left\{\begin{array}{l}
x^{2^{m}+1}+y^{2^{m}+1}+z^{2^{m}+1}=0 \\
x^{2^{k}+1}+y^{2^{k}+1}+z^{2^{k}+1}=0
\end{array}\right.
$$

In the case $x y z=0$, we may assume $z=0$. Then (17) has $M_{2}$ solutions. Hence (17) has $3 M_{2}-2$ solutions satisfying $x y z=0$.

In the case $x y z \neq 0$. Assume $z=1$. By (17) we get

$$
\left(x^{2^{k}+1}+1\right)^{2^{m}+1}=\left(y^{2^{k}+1}\right)^{2^{m}+1}=\left(y^{2^{m}+1}\right)^{2^{k}+1}=\left(x^{2^{m}+1}+1\right)^{2^{k}+1} .
$$

Therefore we have Therefore we get

$$
\left(x^{2^{m+k}}+x\right)\left(x^{2^{m}}+x^{2^{k}}\right)=0 .
$$

Since $\operatorname{gcd}(m+k, n)=\operatorname{gcd}(m-k, n)=d^{\prime}$, we have $x \in \mathbb{F}_{2^{d^{\prime}}}$.

- If $d^{\prime}=d$, then (17) with $z=1$ reduces to $x^{2}+y^{2}+1=0$, i.e. $x+y+1=0$ which has $2^{d}-2$ solutions in $\mathbb{F}_{2^{d}}^{2}$ satisfying $x y \neq 0$. Hence

$$
M_{3}=3 M_{2}-2+\left(2^{d}-2\right) \cdot\left(2^{n}-1\right)=2^{n+d}+2^{n}-2^{d} .
$$

- If $d^{\prime}=2 d$, then (17) with $z=1$ reduces to

$$
x^{2^{d}+1}+y^{2^{d}+1}+1=0
$$


with $(x, y) \in \mathbb{F}_{2^{2 d}}^{*} \times \mathbb{F}_{2^{2 d}}^{*}$. Note that (18) defines a Hermitian curve on $\mathbb{F}_{2^{2 d}}$ which has $2^{3 d}+1$ rational (projective) points (see [?]). Since (18) has $2^{d}+1$ infinite points: $\left(1: t_{1}: 0\right)$ with $2^{2^{d}+1}=1$, and $2\left(2^{d}+1\right)$ solutions satisfying $x y=0:\left(t_{1}, 0\right),\left(0, t_{2}\right)$ with $t_{1}^{d^{d}+1}=t_{2}^{2^{d}+1}=1$, we get

$$
M_{3}=3 M_{2}-2+\left(\left(2^{3 d}+1\right)-3\left(2^{d}+1\right)\right) \cdot\left(2^{n}-1\right)=2^{n+3 d}+2^{n}-2^{3 d} .
$$

Remark. For the case $d^{\prime}=2 d, \sum_{\alpha \in \mathbb{F}_{2} m, \beta \in \mathbb{F}_{q}} T(\alpha, \beta)^{3}$ can also be determined, but we do not need this result.

In the case $d^{\prime}=2 d$, we could determine the explicit values of $T(\alpha, \beta)$. To this end we need to study a class of Artin-Schreier curves. A similar technique has been employed in Coulter [3], Theorem 5.2.

Lemma 7. Suppose $\left(\alpha^{\prime}, \beta\right) \in \mathbb{F}_{q}^{2} \backslash\{0,0\}$ such that $\operatorname{Tr}_{m}^{n}\left(\alpha^{\prime}\right)=\alpha$ and $d^{\prime}=2 d$. Let $N$ be the number of $\mathbb{F}_{q}$-rational (affine) points on the curve

$$
\alpha^{\prime} x^{2^{m}+1}+\beta x^{2^{k}+1}=y^{2^{d}}+y .
$$

Then

$$
N=2^{n}+\left(2^{d}-1\right) \cdot T(\alpha, \beta) .
$$

Proof. We get that

$$
\begin{aligned}
& q N=\sum_{\omega \in \mathbb{F}_{q}} \sum_{x, y \in \mathbb{F}_{q}}(-1)^{\operatorname{Tr}_{1}^{n}\left(\omega\left(\alpha^{\prime} x^{2^{m}+1}+\beta x^{2^{k}+1}+y^{2^{d}}+y\right)\right)} \\
& =q^{2}+\sum_{\omega \in \mathbb{F}_{q}^{*}} \sum_{x \in \mathbb{F}_{q}}(-1)^{\operatorname{Tr}_{1}^{n}\left(\omega\left(\alpha^{\prime} x^{2^{m}+1}+\beta x^{2^{k}+1}\right)\right)} \sum_{y \in \mathbb{F}_{q}}(-1)^{\operatorname{Tr}_{1}^{n}\left(y^{2^{d}}\left(\omega^{2^{d}}+\omega\right)\right)} \\
& =q^{2}+q \sum_{\omega \in \mathbb{F}_{q_{0}}^{*}} \sum_{x \in \mathbb{F}_{q}}(-1)^{\operatorname{Tr}_{1}^{n}\left(\omega\left(\alpha^{\prime} x^{2^{m}+1}+\beta x^{2^{k}+1}\right)\right)} \\
& =q^{2}+q \sum_{\omega \in \mathbb{F}_{q_{0}}^{*}} \sum_{x \in \mathbb{F}_{q}}(-1)^{\operatorname{Tr}_{1}^{m}\left(\omega \alpha x^{2^{m}+1}\right)+\operatorname{Tr}_{1}^{n}\left(\omega \beta x^{2^{k}+1}\right)} \\
& =q^{2}+q \sum_{\omega \in \mathbb{F}_{q_{0}}^{*}} T(\omega \alpha, \omega \beta)
\end{aligned}
$$

where the 3-rd equality follows from that the inner sum is zero unless $\omega^{2^{d}}+\omega=0$, i.e. $\omega \in \mathbb{F}_{q_{0}}$ and the 4-th equality follows from $\omega \alpha^{\prime} x^{p^{m}+1} \in \mathbb{F}_{p^{m}}$.

For any $\omega \in \mathbb{F}_{q_{0}}^{*}$, choose $t \in \mathbb{F}_{2^{2 d}}$ such that $t^{2^{d}+1}=\omega$. Then $t^{2^{m}+1}=t^{2^{k}+1}=\omega$. As a consequence $T(\omega \alpha, \omega \beta)=T(\alpha, \beta)$. Hence $N=2^{n}+\left(2^{d}-1\right) \cdot T(\alpha, \beta)$. 
Now we give an explicit evaluation of $T(\alpha, \beta)$ in the case $d^{\prime}=2 d$.

Lemma 8. Assumptions as in Lemma 7. Then

$$
T(\alpha, \beta)= \begin{cases}-2^{m}, & \text { if } r_{\alpha, \beta}=s \\ 2^{m+d}, & \text { if } r_{\alpha, \beta}=s-2 \\ -2^{m+2 d}, & \text { if } r_{\alpha, \beta}=s-4\end{cases}
$$

Proof. Consider the $\mathbb{F}_{q}$-rational (affine) points on the Artin-Schreier curve in Lemma 7. It is easy to verify that $(0, y)$ with $y \in \mathbb{F}_{q_{0}}$ are exactly the points on the curve with $x=0$. If $(x, y)$ with $x \neq 0$ is a point on this curve, then so are $\left(t x, t^{2^{d}+1} y\right)$ with $t^{2^{2 d}-1}=1\left(\right.$ note that $2^{m}+1 \equiv 2^{k}+1 \equiv 2^{d}+1\left(\bmod 2^{2 d}-1\right)$ since $m / d$ and $k / d$ are both odd by (10) $)$. In total, we have

$$
2^{n}+\left(2^{d}-1\right) T(\alpha, \beta)=N \equiv 2^{d} \quad\left(\bmod 2^{2 d}-1\right)
$$

which yields

$$
T(\alpha, \beta) \equiv 1 \quad\left(\bmod 2^{d}+1\right)
$$

Obviously $T(\alpha, \beta) \neq 0$. We only consider the case $r_{\alpha, \beta}=s$. The other cases are similar. In this case $T(\alpha, \beta)= \pm 2^{m}$. Assume $T(\alpha, \beta)=2^{m}$. Then $2^{d}+1 \mid 2^{m}-1$ which contradicts to $m / d$ is odd. Therefore $T(\alpha, \beta)=-2^{m}$.

Remark. Applying Lemma 8 to Lemma 7, we could determine the number of rational points on the curve (19).

\section{Exponential Sums $T(\alpha, \beta)$ and Cyclic Code $\mathcal{C}_{1}$}

Recall $q_{0}^{*}=(-1)^{\frac{q_{0}-1}{2}} q_{0}$. In this section we prove the following results.

Theorem 1. The value distribution of the multi-set $\left\{T(\alpha, \beta) \mid \alpha \in \mathbb{F}_{2^{m}}, \beta \in \mathbb{F}_{q}\right\}$ and the weight distribution of $\mathcal{C}_{1}$ are shown as following (Column 1 is the value of $T(\alpha, \beta)$, Column 2 is the weight of $c(\alpha, \beta)=\left(\operatorname{Tr}_{1}^{m}\left(\alpha \pi^{i\left(2^{m}+1\right)}\right)+\operatorname{Tr}_{1}^{n}\left(\beta \pi^{i\left(2^{k}+1\right)}\right)\right)_{i=0}^{q-2}$ and Column 3 is the corresponding multiplicity).

(i). For the case $d^{\prime}=d$, 


\begin{tabular}{|c|c|c|}
\hline value & weight & multiplicity \\
\hline $2^{m}$ & $2^{n-1}-2^{m-1}$ & $\frac{2^{d-1}\left(2^{m}-1\right)\left(2^{n}+2^{m+1}+1\right)}{2^{d}+1}$ \\
\hline$-2^{m}$ & $2^{n-1}+2^{m-1}$ & $\frac{2^{d-1}\left(2^{m}-1\right)\left(2^{n}-2^{n-d+1}+1\right)}{2^{d}-1}$ \\
\hline$-2^{m+d}$ & $2^{n-1}+2^{m+d-1}$ & $\frac{\left(2^{m-d}-1\right)\left(2^{n}-1\right)}{2^{2 d}-1}$ \\
\hline 0 & $2^{n-1}$ & $2^{m-d}\left(2^{n}-1\right)$ \\
\hline $2^{n}$ & 0 & 1 \\
\hline
\end{tabular}

(ii). For the case $d^{\prime}=2 d$,

\begin{tabular}{|c|c|c|}
\hline value & weight & multiplicity \\
\hline$-2^{m}$ & $2^{n-1}+2^{m-1}$ & $\frac{2^{3 d}\left(2^{m}-1\right)\left(2^{n}-2^{n-2 d}-2^{n-3 d}+2^{m}-2^{m-d}+1\right)}{\left(2^{d}+1\right)\left(2^{2 d}-1\right)}$ \\
\hline $2^{m+d}$ & $2^{n-1}-2^{m+d-1}$ & $\frac{2^{d}\left(2^{n}-1\right)\left(2^{m}+2^{m-d}+2^{m-2 d}+1\right)}{\left(2^{d}+1\right)^{2}}$ \\
\hline$-2^{m+2 d}$ & $2^{n-1}-2^{m+2 d-1}$ & $\frac{\left(2^{m-d}-1\right)\left(2^{n}-1\right)}{\left(2^{d}+1\right)\left(2^{2 d}-1\right)}$ \\
\hline $2^{m}$ & 0 & 1 \\
\hline
\end{tabular}

Proof. Define

$$
N_{i}=\left\{(\alpha, \beta) \in \mathbb{F}_{2^{m}} \times \mathbb{F}_{q} \backslash\{(0,0)\} \mid r_{\alpha, \beta}=s-i\right\} .
$$

Then $n_{i}=\left|N_{i}\right|$.

According to Lemma1 (setting $F(X)=X H_{\alpha, \beta} X^{T}=\operatorname{Tr}_{d}^{m}\left(\alpha x^{2^{m}+1}\right)+\operatorname{Tr}_{d}^{n}\left(\beta x^{2^{k}+1}\right)$ ), we define that for $\varepsilon= \pm 1$ and $0 \leq i \leq s-1$,

$$
N_{i, \varepsilon}=\left\{(\alpha, \beta) \in \mathbb{F}_{2^{m}} \times \mathbb{F}_{q} \backslash\{(0,0)\} \mid T(\alpha, \beta)=\varepsilon \cdot 2^{m+\frac{i d}{2}}\right\} .
$$

and $n_{i, \varepsilon}=\left|N_{i, \varepsilon}\right|$. Then by Lemma 1 we have $N_{0}=N_{0,1} \bigcup N_{0,-1}$ and $n_{0}=$ $n_{0,1}+n_{0,-1}$. But for general $i, N_{i} \neq N_{i, 1} \bigcup N_{i,-1}$ and $n_{i} \neq n_{i, 1}+n_{i,-1}$.

Meanwhile we define $\omega$ to be the number of $(\alpha, \beta) \in \mathbb{F}_{2^{m}} \times \mathbb{F}_{q} \backslash\{(0,0)\}$ such that $T(\alpha, \beta)=0$.

From (11) we know that for each non-zero codeword $c(\alpha, \beta)=\left(c_{0}, \cdots, c_{l-1}\right)$ $\left(l=q-1, c_{i}=\operatorname{Tr}_{1}^{m}\left(\alpha \pi^{\left(2^{m}+1\right) i}\right)+\operatorname{Tr}_{1}^{n}\left(\beta \pi^{\left(2^{k}+1\right) i}\right), 0 \leq i \leq l-1\right.$, and $(\alpha, \beta) \in$ $\left.\mathbb{F}_{2^{m}} \times \mathbb{F}_{q}\right)$, the Hamming weight of $c(\alpha, \beta)$ is

$$
w_{H}(c(\alpha, \beta))=2^{n-1}-\frac{1}{2} \cdot T(\alpha, \beta) .
$$


(i). For the case $d^{\prime}=d$, by Lemma 1 and Lemma 3 we know that the possible values of $T(\alpha, \beta)$ for $(\alpha, \beta) \in \mathbb{F}_{2^{m}} \times \mathbb{F}_{q} \backslash\{(0,0)\}$ is $0, \pm 2^{m}, \pm 2^{m+d}$. Moreover from Lemma 6 we have

$$
\begin{gathered}
\left(n_{0,1}-n_{0,-1}\right)+2^{d}\left(n_{2,1}-n_{2,-1}\right)=2^{m}\left(2^{m}-1\right) \\
\left(n_{0,1}+n_{0,-1}\right)+2^{2 d}\left(n_{2,1}+n_{2,-1}\right)=2^{n}\left(2^{m}-1\right) \\
\left(n_{0,1}-n_{0,-1}\right)+2^{3 d}\left(n_{2,1}-n_{2,-1}\right)=-2^{3 m}+2^{n+d}+2^{n}-2^{d} .
\end{gathered}
$$

In addition, by Lemma 1 and Lemma 3 we have

$$
\omega+\left(n_{0,1}+n_{0,-1}\right)+\left(n_{2,1}+n_{2,-1}\right)=2^{3 m}-1 .
$$

and

$$
n_{0,1}+n_{0,-1}=n_{0}=\frac{\left(2^{n+2 d}-2^{n+d}-2^{n}+2^{m+2 d}-2^{m+d}+2^{2 d}\right)\left(2^{m}-1\right)}{2^{2 d}-1} .
$$

Combining (21) - (25) we get the result.

(iii). For the case $d^{\prime}=2 d$, by Lemma 8 we have

$$
\omega=n_{0,1}=n_{2,-1}=n_{4,1}=0 .
$$

Combining Lemma 3, Lemma 6, Lemma (8) and (26) we have

$$
\begin{gathered}
n_{0,-1}+n_{2,1}+n_{4,-1}=2^{3 m}-1 \\
-n_{0,-1}+2^{d} \cdot n_{2,1}-2^{2 d} \cdot n_{4,-1}=2^{m}\left(2^{m}-1\right) \\
n_{0,-1}+2^{2 d} \cdot n_{2,1}+2^{4 d} \cdot n_{4,-1}=2^{m}\left(2^{n+d}+2^{n}-2^{m}-2^{d}\right) .
\end{gathered}
$$

Solving the system of equations consisting of (27)-(29) yields the result.

The weight distribution of $\mathcal{C}_{1}$ is derived from the value distribution of $T(\alpha, \beta)$ and (20). 


\section{Results on Correlation Distribution of Sequences and Cyclic Code $\mathcal{C}_{2}$}

Recall $\phi_{\alpha, \beta}(x)$ in the proof of Lemma 3 and $N_{i, \varepsilon}$ in the proof of Theorem 1 . Finally we will determine the value distribution of $S(\alpha, \beta, \gamma)$, the correlation distribution among sequences in $\mathcal{F}$ defined in (5) and the weight distribution of $\mathcal{C}_{2}$ defined in Section 1.

The following lemma, which has been stated for the case $p$ odd in [30] Lemma 5 , is also valid for $p=2$.

Lemma 9. (i). The sequences in $\mathcal{F}$ are all maximal with length $q-1$.

(i). For any given $\alpha \in \mathbb{F}_{2^{m}}^{*}$, when $\beta$ runs through $\mathbb{F}_{q}$, the distribution of $T(\alpha, \beta)$ is the same as $T(1, \beta)$.

(ii). For any given $\gamma \in \mathbb{F}_{q}^{*}$, when $(\alpha, \beta)$ runs through $\mathbb{F}_{p^{m}} \times \mathbb{F}_{q}$, the distribution of $S(\alpha, \beta, \gamma)$ is the same as $S(\alpha, \beta, 1)$.

(iii). Suppose $m / d$ or $k / d$ is even. If $\beta$ runs through $\mathbb{F}_{q}^{*}$, then $C_{\beta}$ runs through the sequences in $\mathcal{F}$ exactly $\left(2^{m}+1\right)$ times.

We are now ready to give the value distribution of $S(\alpha, \beta, \gamma)$ and weight distribution of $\mathcal{C}_{2}$.

Theorem 2. The value distribution of the multi-set $\left\{S(\alpha, \beta, \gamma) \mid \alpha \in \mathbb{F}_{2^{m}},(\beta, \gamma) \in \mathbb{F}_{q}^{2}\right\}$ and the weight distribution of $\mathcal{C}_{2}$ are shown as following (Column 1 is the value of $S(\alpha, \beta, \gamma)$, Column 2 is the weight of $c(\alpha, \beta, \gamma)=\left(\operatorname{Tr}_{1}^{m}\left(\alpha \pi^{i\left(2^{m}+1\right)}\right)+\operatorname{Tr}_{1}^{n}\left(\beta \pi^{i\left(2^{k}+1\right)}+\gamma \pi^{i}\right)\right)_{i=0}^{q-2}$ and Column 3 is the corresponding multiplicity).

(i). For the case $d^{\prime}=d$,

\begin{tabular}{|c|c|c|}
\hline value & weight & multiplicity \\
\hline $2^{m}$ & $2^{n-1}-2^{m-1}$ & $\frac{2^{m-1}\left(2^{n}-1\right)\left(2^{n+2 d}-2^{n+d}-2^{n}+2^{m+2 d}-2^{m+d}+2^{2 d}\right)}{2^{2 d}-1}$ \\
\hline$-2^{m}$ & $2^{n-1}+2^{m-1}$ & $\frac{2^{m-1}\left(2^{m}-1\right)^{2}\left(2^{n+2 d}-2^{n+d}-2^{n}+2^{m+2 d}-2^{m+d}+2^{2 d}\right)}{2^{2 d}-1}$ \\
\hline $2^{m+d}$ & $2^{n-1}-2^{m+d-1}$ & $\frac{2^{m-d-1}\left(2^{m-d}+1\right)\left(2^{m+d}-1\right)\left(2^{n}-1\right)}{2^{2 d}-1}$ \\
\hline$-2^{m+d}$ & $2^{n-1}+2^{m+d-1}$ & $\frac{2^{m-d-1}\left(2^{m-d}-1\right)\left(2^{m+d}-1\right)\left(2^{n}-1\right)}{2^{2 d}-1}$ \\
\hline 0 & $2^{n-1}$ & $\left(2^{3 m-d}-2^{n-2 d}+1\right)\left(2^{n}-1\right)$ \\
\hline $2^{n}$ & 0 & 1 \\
\hline
\end{tabular}


(ii). For the case $d^{\prime}=2 d$,

\begin{tabular}{|c|c|c|}
\hline value & weight & multiplicity \\
\hline $2^{m}$ & $2^{n-1}-2^{m-1}$ & $\frac{2^{m+3 d-1}\left(2^{n}-1\right)\left(2^{n}-2^{n-2 d}-2^{n-3 d}+2^{m}-2^{m-d}+1\right)}{\left(2^{d}+1\right)\left(2^{2 d}-1\right)}$ \\
\hline$-2^{m}$ & $2^{n-1}+2^{m-1}$ & $\frac{2^{m+3 d-1}\left(2^{m}-1\right)^{2}\left(2^{n}-2^{n-2 d}-2^{n-3 d}+2^{m}-2^{m-d}+1\right)}{\left(2^{d}+1\right)\left(2^{2 d}-1\right)}$ \\
\hline $2^{m+d}$ & $2^{n-1}-2^{m+d-1}$ & $\frac{2^{m-1}\left(2^{m-d}+1\right)\left(2^{n}-1\right)\left(2^{m}+2^{m-d}+2^{m-2 d}+1\right)}{\left(2^{d}+1\right)^{2}}$ \\
\hline$-2^{m+d}$ & $2^{n-1}+2^{m+d-1}$ & $\frac{2^{m-1}\left(2^{m-d}-1\right)\left(2^{n}-1\right)\left(2^{m}+2^{m-d}+2^{m-2 d}+1\right)}{\left(2^{d}+1\right)^{2}}$ \\
\hline $2^{m+2 d}$ & $2^{n-1}-2^{m+2 d-1}$ & $\frac{2^{m-2 d-1}\left(2^{m-2 d}+1\right)\left(2^{m-d}-1\right)\left(2^{n}-1\right)}{\left(2^{d}+1\right)\left(2^{2 d}-1\right)}$ \\
\hline$-2^{m+2 d}$ & $2^{n-1}+2^{m+2 d-1}$ & $\left(2^{n}-1\right)\left(2^{3 m-d}-2^{3 m-2 d}+2^{3 m-3 d}-2^{3 m-4 d}+2^{3 m-5 d}+2^{n-d}-2^{n-2 d+1}+2^{n-3 d}-2^{n-4 d}+1\right)$ \\
\hline 0 & $2^{n-1}$ & $\left(2^{d}+1\right)\left(2^{2 d}-1\right)$ \\
\hline $2^{m}$ & 0 & \\
\hline
\end{tabular}

Proof. Define

$$
\Xi=\left\{(\alpha, \beta, \gamma) \in \mathbb{F}_{q}^{3} \mid S(\alpha, \beta, \gamma)=0\right\}
$$

and $\xi=|\Xi|$.

Recall $n_{i}, H_{\alpha, \beta}, r_{\alpha, \beta}, A_{\gamma}$ in Section 1 and $N_{i, \varepsilon}, n_{i, \varepsilon}$, in the proof of Lemma 3, From Lemma 2, if $(\alpha, \beta) \in N_{i, \varepsilon}$, then the number of $\gamma \in \mathbb{F}_{q}$ such that $S(\alpha, \beta, \gamma)=$ 0 is $q_{0}^{s}-q_{0}^{i}$. From Lemma 3 and Theorem 1 we know that

- if $d^{\prime}=d$ and $(\alpha, \beta) \neq(0,0)$, then $r_{\alpha, \beta}=s-i$ for some $i \in\{0,1,2\}$. By Lemma 1 we have

$$
\xi=2^{n}-1+\left(2^{n}-2^{n-2 d}\right) n_{2}=\left(2^{3 m-d}-2^{n-2 d}+1\right)\left(2^{n}-1\right) .
$$

- if $d^{\prime}=2 d$, similarly we have

$$
\begin{gathered}
\xi=2^{n}-1+\left(2^{n}-2^{n-2 d}\right) n_{2,1}+\left(2^{n}-2^{n-4 d}\right) n_{4,-1} \\
=\left(2^{n}-1\right)\left(2^{3 m-d}-2^{3 m-2 d}+2^{3 m-3 d}-2^{3 m-4 d}+2^{3 m-5 d}\right. \\
\left.\quad+2^{n-d}-2^{n-2 d+1}+2^{n-3 d}-2^{n-4 d}+1\right) .
\end{gathered}
$$

From (11) we know that for each non-zero codeword $c(\alpha, \beta, \gamma)=\left(c_{0}, \cdots, c_{n-1}\right)$ $\left(n=2^{n}-1, c_{i}=\operatorname{Tr}_{1}^{m}\left(\alpha \pi^{\left(2^{m}+1\right) i}\right)+\operatorname{Tr}_{1}^{n}\left(\beta \pi^{\left(2^{k}+1\right) i}+\gamma \pi^{i}\right), 0 \leq i \leq q-2\right.$, and $(\alpha, \beta, \gamma) \in$ 
$\left.\mathbb{F}_{2^{m}} \times \mathbb{F}_{q}^{2}\right)$, the Hamming weight of $c(\alpha, \beta, \gamma)$ is

$$
w_{H}(c(\alpha, \beta, \gamma))=p^{n-1}-\frac{1}{2} \cdot S(\alpha, \beta, \gamma)
$$

Combining Lemma 1 and Theorem 1 we get the value distribution of $S(\alpha, \beta, \gamma)$. As a consequence we have the weight distribution of $\mathcal{C}_{2}$ from (32).

Theorem 3. The collection $\mathcal{F}$ defined in (5) is a family of p-ary sequences with period $q-1$.

(i). If $m / d$ is even, then $\mathcal{F}$ has family size $2^{3 m}+2^{m}-1$ and correlation distribution as follows.

\begin{tabular}{|c|c|}
\hline values & multiplicity \\
\hline $2^{m}-1$ & $\left(2^{4 n+2 d-1}-2^{4 n+d-1}-2^{4 n-1}+2^{7 m+2 d-1}-2^{7 m+d-1}+2^{3 n+2 d-1}-2^{5 m+2 d}+2^{5 m+d}+2^{5 m}\right.$ \\
\hline$-2^{m}-1$ & $\left.-2^{2 n+2 d+1}+2^{2 n+d+1}+2^{2 n}-2^{3 m+2 d}-2^{3 m}+2^{n+2 d}-2^{n+d+1}+2^{m+2 d+1}-2^{m+d}-2^{2 d}+2^{d}\right) /\left(2^{2 d}-1\right)$ \\
\hline $2^{m+d}-1$ & $\left(2^{4 n+2 d-1}-2^{4 n+d-1}-2^{4 n-1}-2^{7 m+2 d-1}+2^{7 m+d-1}+2^{7 m}+2^{3 n+2 d-1}-2^{3 n}-2^{5 m+2 d+1}+2^{5 m+d}+2^{5 m+1}\right.$ \\
\hline$-2^{m+d}-1$ & $\left.+2^{2 n+2 d}-2^{2 n+1}-2^{3 m+d+1}+2^{n+2 d}+2^{n+d}+2^{n}-2^{m}-2^{2 d}+2^{d}+2\right) /\left(2^{2 d}-1\right)$ \\
\hline-1 & $\frac{2^{m-d}\left(2^{m-d}+1\right)\left(2^{m+d}-1\right)\left(2^{5 m-1}-2^{n}-2^{m}+1\right)}{2^{2 d}-1}$ \\
\hline $2^{n}-1$ & $\left(2^{4 n-d-1}-2^{7 m-1}-2^{7 m-2 d-1}+2^{3 n-d-1}-2^{5 m-d}+2^{2 n}-2^{2 n-d}+2^{2 n-2 d}\right.$ \\
$2^{4 n-d}-2^{7 m-2 d}+2^{5 m}-2^{5 m-d+1}-2^{2 n-d+1}+2^{2 n-2 d+1}$ \\
$+2^{3 m-d+1}+2^{3 m-2 d+1}-2^{n+1}-2^{n-2 d+1}-2^{m+1}+2^{m-d+1}+2$ \\
\hline
\end{tabular}

(ii). If $k / d$ is even, then $\mathcal{F}$ has family size $2^{3 m}+2^{m}$ and correlation distribution as follows. 


\begin{tabular}{|c|c|}
\hline values & multiplicity \\
\hline $2^{m}-1$ & $\frac{2^{4 n+2 d-1}-2^{4 n+d-1}-2^{4 n-1}+2^{7 m+2 d-1}-2^{7 m+d-1}+2^{3 n+2 d-1}-2^{2 n+2 d}+2^{2 n+d}+2^{2 n}-2^{3 m+2 d}+2^{3 m+d}-2^{n+2 d}}{2^{2 d}-1}$ \\
\hline$-2^{m}-1$ & $\left(2^{4 n+2 d-1}-2^{4 n+d-1}-2^{4 n-1}-2^{7 m+2 d-1}+2^{7 m+d-1}+2^{7 m}+2^{3 n+2 d-1}-2^{3 n}\right.$ \\
\hline $2^{m+d}-1$ & $\left.-2^{5 m+2 d}+2^{5 m}+2^{2 n+d}-2^{3 m+d}-2^{3 m}+2^{n}+2^{m+2 d}-2^{m}\right) /\left(2^{2 d}-1\right)$ \\
\hline$-2^{m+d}-1$ & $\frac{2^{n-d}\left(2^{m-d}+1\right)\left(2^{m+d}-1\right)\left(2^{2 n-1}-1\right)}{2^{2 d}-1}$ \\
\hline-1 & $\frac{2^{n-d}\left(2^{m-d}-1\right)\left(2^{m+d}-1\right)\left(2^{2 n-1}-1\right)}{2^{2 d}-1}$ \\
\hline $2^{n}-1$ & $2^{4 n-d}-2^{7 m-2 d}+2^{5 m}-2^{2 n-d+1}+2^{3 m-2 d+1}-2^{m+1}$ \\
\hline
\end{tabular}

(iii). If $m / d$ and $k / d$ are both odd(that is, $\left.d^{\prime}=2 d\right)$, then $\mathcal{F}$ has family size $2^{3 m}$ and correlation distribution as follows.

\begin{tabular}{|c|c|}
\hline values & multiplicity \\
\hline $2^{m}$ & $\frac{2^{2 n+3 d-1}\left(2^{n}-2\right)\left(2^{n}-2^{n-2 d}-2^{n-3 d}+2^{m}-2^{m-d}+1\right)}{\left(2^{d}+1\right)\left(2^{2 d}-1\right)}$ \\
\hline$-2^{m}$ & $\frac{2^{3 m+3 d}\left(2^{3 m-1}-2^{n}+1\right)\left(2^{n}-2^{n-2 d}-2^{n-3 d}+2^{m}-2^{m-d}+1\right)}{\left(2^{d}+1\right)\left(2^{2 d}-1\right)}$ \\
\hline $2^{m+d}$ & $\frac{2^{3 m}\left(2^{2 n-d-1}+2^{3 m-1}-2^{n-d}-2^{m}+2^{d}\right)\left(2^{m}+2^{m-d}+2^{m-2 d}+1\right)}{\left(2^{d}+1\right)^{2}}$ \\
\hline$-2^{m+d}$ & $\frac{2^{2 n-1}\left(2^{m-d}-1\right)\left(2^{n}-2\right)\left(2^{m}+2^{m-d}+2^{m-2 d}+1\right)}{\left(2^{d}+1\right)^{2}}$ \\
\hline $2^{m+2 d}$ & $\frac{2^{2 n-2 d-1}\left(2^{m-2 d}+1\right)\left(2^{m-d}-1\right)\left(2^{n}-2\right)}{\left(2^{d}+1\right)\left(2^{2 d}-1\right)}$ \\
\hline$-2^{m+2 d}$ & $\frac{2^{3 m}\left(2^{m-d}-1\right)\left(2^{2 n-2 d-1}-2^{3 m-2 d-1}-2^{n-2 d}+2^{m-2 d}+1\right)}{\left(2^{d}+1\right)\left(2^{2 d}-1\right)}$ \\
\hline 0 & $2^{3 m}\left(2^{n}-2\right)\left(2^{3 m-d}-2^{3 m-2 d}+2^{3 m-3 d}-2^{3 m-4 d}+2^{3 m-5 d}+2^{n-d}-2^{n-2 d+1}+2^{n-3 d}-2^{n-4 d}+1\right)$ \\
\hline $2^{m}$ & $2^{3 m}$ \\
\hline
\end{tabular}

Proof. For any possible value $\kappa$ and $1 \leq i, j \leq 3$, define $M_{\kappa}\left(\mathcal{F}_{i}, \mathcal{F}_{j}\right)$ to be the frequency of $\kappa$ in correlation values between two sequences in $\mathcal{F}_{i}$ and $\mathcal{F}_{j}$ by any shift, respectively. Then the correlation distribution of sequences in $\mathcal{F}$ could be obtained if we can calculate all of the $M_{\kappa}\left(\mathcal{F}_{i}, \mathcal{F}_{j}\right)$. We will deal with it case by case.

- The correlation function between $a_{\alpha_{1}, \beta_{1}}$ and $a_{\alpha_{2}, \beta_{2}}$ by a shift $\tau(0 \leq \tau \leq q-2)$ 
is

$$
\begin{aligned}
& C_{\left(\alpha_{1}, \beta_{1}\right),\left(\alpha_{2}, \beta_{2}\right)}(\tau)=\sum_{\lambda=0}^{q-2}(-1)^{a_{\alpha_{1}, \beta_{1}}(\lambda)-a_{\alpha_{2}, \beta_{2}}(\lambda+\tau)} \\
& \quad=\sum_{\lambda=0}^{q-2}(-1)^{\operatorname{Tr}_{1}^{m}\left(\alpha_{1} \pi^{\lambda\left(2^{m}+1\right)}\right)+\operatorname{Tr}_{1}^{n}\left(\beta_{1} \pi^{\lambda\left(2^{k}+1\right)}+\pi^{\lambda}\right)-\operatorname{Tr}_{1}^{m}\left(\alpha_{2} \pi^{(\lambda+\tau)\left(2^{m}+1\right)}\right)-\operatorname{Tr}_{1}^{n}\left(\beta \pi^{(\lambda+\tau)\left(2^{k}+1\right)}+\pi^{\lambda+\tau}\right)} \\
& =S\left(\alpha^{\prime}, \beta^{\prime}, \gamma^{\prime}\right)-1
\end{aligned}
$$

where

$$
\alpha^{\prime}=\alpha_{1}-\alpha_{2} \pi^{\tau\left(2^{m}+1\right)}, \quad \beta^{\prime}=\beta_{1}-\beta_{2} \pi^{\tau\left(2^{k}+1\right)}, \quad \gamma^{\prime}=1-\pi^{\tau} .
$$

Fix $\left(\alpha_{2}, \beta_{2}\right) \in \mathbb{F}_{2^{m}} \times \mathbb{F}_{q}$, when $\left(\alpha_{1}, \beta_{1}\right)$ runs through $\mathbb{F}_{2^{m}} \times \mathbb{F}_{q}$ and $\tau$ takes values from 0 to $q-2,\left(\alpha^{\prime}, \beta^{\prime}, \gamma^{\prime}\right)$ runs through $\mathbb{F}_{2^{m}} \times \mathbb{F}_{q} \times\left\{\mathbb{F}_{q} \backslash\{1\}\right\}$ exactly one time.

For any possible value $\kappa$ of $S(\alpha, \beta, \gamma)$, define

$$
\begin{gathered}
s_{\kappa}=\#\left\{(\alpha, \beta, \gamma) \in \mathbb{F}_{2^{m}} \times \mathbb{F}_{q} \times \mathbb{F}_{q} \mid S(\alpha, \beta, \gamma)=\kappa+1\right\} \\
s_{\kappa}^{1}=\#\left\{(\alpha, \beta) \in \mathbb{F}_{2^{m}} \times \mathbb{F}_{q} \mid S(\alpha, \beta, 1)=\kappa+1\right\}
\end{gathered}
$$

and

$$
t_{\kappa}=\#\left\{(\alpha, \beta) \in \mathbb{F}_{2^{m}} \times \mathbb{F}_{q} \mid T(\alpha, \beta)=\kappa+1\right\} .
$$

By Lemma 9 we have

$$
s_{\kappa}^{1}=\frac{1}{2^{n}-1} \times\left(s_{\kappa}-t_{\kappa}\right) .
$$

Hence we get

$$
M_{\kappa}\left(\mathcal{F}_{1}, \mathcal{F}_{1}\right)=2^{3 m} \cdot\left(s_{\kappa}-s_{\kappa}^{1}\right)=2^{3 m} \cdot\left(\frac{2^{n}-2}{2^{n}-1} \cdot s_{\kappa}+\frac{1}{2^{n}-1} \cdot t_{\kappa}\right) .
$$

- For the case $m / d$ or $k / d$ is even. The cross correlation function between $a_{\alpha_{1}, \beta_{1}}$ and $a_{\beta_{2}}$ by a shift $\tau(0 \leq \tau \leq q-2)$ is

$$
\begin{aligned}
& C_{\left(\alpha_{1}, \beta_{1}\right), \beta_{2}}(\tau)=\sum_{\lambda=0}^{q-2}(-1)^{a_{\alpha_{1}, \beta_{1}}(\lambda)-a_{\beta_{2}}(\lambda+\tau)}
\end{aligned}
$$

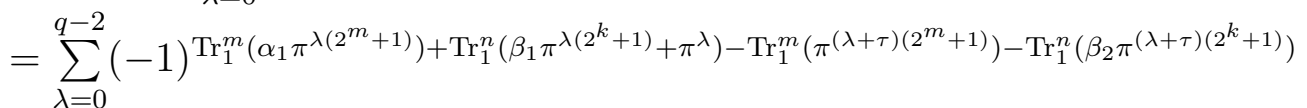

$$
\begin{aligned}
& =S\left(\alpha^{\prime}, \beta^{\prime}, 1\right)-1
\end{aligned}
$$


where $\alpha^{\prime}=\alpha_{1}-\pi^{\tau\left(2^{m}+1\right)}, \beta^{\prime}=\beta_{1}-\beta_{2} \pi^{\tau\left(2^{k}+1\right)}$.

Fix $0 \leq \tau \leq q-2$ and $\beta_{2}=\pi^{i}$ for some $0 \leq i \leq 2^{m}-2$, when $\left(\alpha_{1}, \beta_{1}\right)$ runs through $\mathbb{F}_{2^{m}} \times \mathbb{F}_{q},\left(\alpha^{\prime}, \beta^{\prime}\right)$ runs through $\mathbb{F}_{2^{m}} \times \mathbb{F}_{q}$ exactly one time. By (34) and (35) we get

$$
M_{\kappa}\left(\mathcal{F}_{1}, \mathcal{F}_{2}\right)=M_{\kappa}\left(\mathcal{F}_{2}, \mathcal{F}_{1}\right)=(q-1)\left(2^{m}-1\right) \cdot s_{\kappa}^{1}=\left(2^{m}-1\right)\left(s_{\kappa}-t_{\kappa}\right) .
$$

- For the case $k / d$ is even. The cross correlation function between $a_{\alpha_{1}, \beta_{1}}$ and $a$ by a shift $\tau(0 \leq \tau \leq q-2)$ is

$$
\begin{aligned}
& C_{\left(\alpha_{1}, \beta_{1}\right)}(\tau)=\sum_{\lambda=0}^{q-2}(-1)^{a_{\alpha_{1}, \beta_{1}}(\lambda)-a(\lambda+\tau)} \\
& =\sum_{\lambda=0}^{q-2}(-1)^{\operatorname{Tr}_{1}^{m}\left(\alpha_{1} \pi^{\lambda\left(2^{m}+1\right)}\right)+\operatorname{Tr}_{1}^{n}\left(\beta_{1} \pi^{\lambda\left(2^{k}+1\right)}+\pi^{\lambda}\right)-\operatorname{Tr}_{1}^{n}\left(\pi^{(\lambda+\tau)\left(2^{k}+1\right)}\right)} \\
& =S\left(\alpha_{1}, \beta^{\prime}, 1\right)-1
\end{aligned}
$$

where $\beta^{\prime}=\beta_{1}-\pi^{\tau\left(2^{k}+1\right)}$.

For fixed $\tau, 0 \leq \tau \leq q-2$, when $\beta_{1}$ runs through $\mathbb{F}_{q}, \beta^{\prime}$ runs through $\mathbb{F}_{q}$ exactly one time. By (34) and (35) we get

$$
M_{\kappa}\left(\mathcal{F}_{1}, \mathcal{F}_{3}\right)=M_{\kappa}\left(\mathcal{F}_{3}, \mathcal{F}_{1}\right)=(q-1) \cdot s_{\kappa}^{1}=s_{\kappa}-t_{\kappa} .
$$

- For the case $m / d$ or $k / d$ is even. The cross correlation function between $a_{\beta_{1}}$ and $a_{\beta_{2}}$ by a shift $\tau(0 \leq \tau \leq q-2)$ is

$$
\begin{aligned}
& C_{\beta_{1}, \beta_{2}}(\tau)=\sum_{\lambda=0}^{q-2}(-1)^{a_{\beta_{1}}(\lambda)-a_{\beta_{2}}(\lambda+\tau)} \\
& =\sum_{\lambda=0}^{q-2}(-1)^{\operatorname{Tr}_{1}^{m}\left(\pi^{\lambda\left(2^{m}+1\right)}\right)+\operatorname{Tr}_{1}^{n}\left(\beta_{1} \pi^{\lambda\left(2^{k}+1\right)}\right)-\operatorname{Tr}_{1}^{m}\left(\pi^{(\lambda+\tau)\left(2^{m}+1\right)}\right)-\operatorname{Tr}_{1}^{n}\left(\beta_{2} \pi^{(\lambda+\tau)\left(2^{k}+1\right)}\right)} \\
& =T\left(\alpha^{\prime}, \beta^{\prime}\right)-1
\end{aligned}
$$

where $\alpha^{\prime}=1-\pi^{\tau\left(2^{m}+1\right)}, \beta^{\prime}=\beta_{1}-\beta_{2} \pi^{\tau\left(2^{k}+1\right)}$.

When $\left(\beta_{1}, \beta_{2}\right)$ runs through $\mathbb{F}_{q} \times \mathbb{F}_{q}$ and $\tau$ takes value from 0 to $q-2$, $\left(\alpha^{\prime}, \beta^{\prime}\right)$ runs through $\mathbb{F}_{2^{m}} \backslash\{1\} \times \mathbb{F}_{q}$ exactly $\left(2^{m}+1\right) \cdot q$ times.

Fix $\beta_{2}=0$. When $\beta_{1}$ runs through $\mathbb{F}_{q}$ and $\tau$ takes value from 0 to $q-2$, $\left(\alpha^{\prime}, \beta^{\prime}\right)$ runs through $\mathbb{F}_{2^{m}} \backslash\{1\} \times \mathbb{F}_{q}$ exactly $\left(2^{m}+1\right)$ times. By symmetry, this statement is also valid if we exchange $\beta_{1}$ and $\beta_{2}$ to each other. 
When $\beta_{1}=\beta_{2}=0$ and $\tau$ takes value from 0 to $q-2$, then $\beta^{\prime}=0$ and $\alpha^{\prime}$ runs through $\mathbb{F}_{2^{m}} \backslash\{1\}$ exactly $\left(2^{m}+1\right)$ times. In this case $\phi_{\alpha^{\prime}, 0}(x)$ defined in (14) is $\alpha^{\prime} x^{2^{m}}$. Hence $T\left(\alpha^{\prime}, 0\right)=2^{n}$ or $-2^{m}$ provided that $\alpha^{\prime}=0$ or not. Define

$$
l_{\kappa}= \begin{cases}1, & \text { if } \kappa=2^{n}-1 \\ 2^{m}-2, & \text { if } \kappa=-2^{m}-1 \\ 0, & \text { otherwise }\end{cases}
$$

Define

$$
t_{\kappa}^{0}=\#\left\{\beta \in \mathbb{F}_{q} \mid T(0, \beta)=\kappa+1\right\} .
$$

Then a routine calculation shows that

$$
t_{\kappa}^{0}=\left\{\begin{array}{cl}
1, & \kappa=2^{n}-1 \\
2^{n}-1, & \kappa=-1 \text { and } k / d \text { is even } \\
\frac{2^{d}\left(2^{n}-1\right)}{2^{d}+1}, & \kappa=2^{m}-1 \text { and } m / d \text { is even } \\
\frac{2^{n}-1}{2^{d}+1}, & \kappa=-2^{m+d}-1 \text { and } m / d \text { is even } \\
0, & \text { otherwise. }
\end{array}\right.
$$

By Inclusion-Exclusion principle, Lemma 9, (36) and (38) we get

$$
\begin{aligned}
M_{\kappa}\left(\mathcal{F}_{2}, \mathcal{F}_{2}\right) & =\left(\frac{1}{2^{m}+1}\right)^{2} \cdot\left(2^{m}+1\right)\left[\left(2^{n}-2\right)\left(\frac{2^{m}-2}{2^{m}-1}\left(t_{\kappa}-t_{\kappa}^{0}\right)+t_{\kappa}^{0}\right)+l_{\kappa}\right] \\
& =\frac{\left(2^{m}-2\right)\left(2^{n}-2\right)}{2^{n}-1} t_{\kappa}+\frac{2^{n}-2}{2^{n}-1} t_{\kappa}^{0}+\frac{1}{2^{m}+1} l_{\kappa} .
\end{aligned}
$$

- For the case $k / d$ is even. The cross correlation function between $a_{\beta} \in \mathcal{F}_{2}$ and $a \in \mathcal{F}_{3}$ by a shift $\tau(0 \leq \tau \leq q-2)$ is

$$
\begin{aligned}
C_{\beta}(\tau) & =\sum_{\lambda=0}^{q-2}(-1)^{a_{\beta}(\lambda)-a(\lambda+\tau)} \\
= & \sum_{\lambda=0}^{q-2}(-1)^{\operatorname{Tr}_{1}^{m}\left(\pi^{\lambda\left(2^{m}+1\right)}\right)+\operatorname{Tr}_{1}^{n}\left(\beta \pi^{\lambda\left(2^{k}+1\right)}\right)-\operatorname{Tr}_{1}^{n}\left(\pi^{(\lambda+\tau)\left(2^{k}+1\right)}\right)} \\
= & T\left(1, \beta^{\prime}\right)-1
\end{aligned}
$$

where $\beta^{\prime}=\beta-\pi^{\tau\left(2^{k}+1\right)}$.

When $\beta$ runs through $\mathbb{F}_{q}^{*}$ and $\tau$ takes value from 0 to $q-2, \beta^{\prime}$ runs through $\mathbb{F}_{q}$ exactly $q-2$ times except for 0 , on which $\beta^{\prime}$ has frequency $q-1$. 
Define

$$
t_{\kappa}^{1}=\#\left\{\beta \in \mathbb{F}_{q} \mid T(1, \beta)=\kappa+1\right\} .
$$

Then from Lemma (9) we have

$$
t_{\kappa}^{1}=\frac{t_{\kappa}-t_{\kappa}^{0}}{2^{m}-1}
$$

Note that $T(1,0)=-2^{m}$. By Lemma 9 we get

$$
\begin{aligned}
M_{\kappa}\left(\mathcal{F}_{2}, \mathcal{F}_{3}\right) & =M_{\kappa}\left(\mathcal{F}_{3}, \mathcal{F}_{2}\right)=\frac{1}{2^{m}+1}\left((q-2) t_{\kappa}^{1}+\delta(\kappa, 0)\right) \\
& =\frac{2^{n}-2}{2^{n}-1}\left(t_{\kappa}-t_{\kappa}^{0}\right)+\frac{1}{2^{m}+1} \delta\left(\kappa,-2^{m}-1\right)
\end{aligned}
$$

where the Hermitian symbol $\delta\left(\kappa,-2^{m}-1\right)=1$ if $\kappa=-2^{m}-1$, and zero otherwise.

- For the case $k / d$ is even. The auto-correlation function of $a \in \mathcal{F}_{3}$ by a shift $\tau(0 \leq \tau \leq q-2)$ is

$$
\begin{aligned}
C(\tau) & =\sum_{\lambda=0}^{q-2}(-1)^{a(\lambda)-a(\lambda+\tau)} \\
& =\sum_{\lambda=0}^{q-2}(-1)^{\operatorname{Tr}_{1}^{n}\left(\pi^{\lambda\left(2^{k}+1\right)}\right)-\operatorname{Tr}_{1}^{n}\left(\pi^{(\lambda+\tau)\left(2^{k}+1\right)}\right)} \\
& =T\left(0,1-\pi^{\tau\left(2^{k}+1\right)}\right)-1 .
\end{aligned}
$$

Since $\operatorname{gcd}\left(2^{k}+1,2^{n}-1\right)=1, C(\tau)=-1$ for $\tau \neq 0$ and $C(0)=q-1$. Then

$$
M_{\kappa}\left(\mathcal{F}_{3}, \mathcal{F}_{3}\right)= \begin{cases}q-2, & \text { if } \kappa=-1 \\ 1, & \text { if } \kappa=q-1 \\ 0, & \text { otherwise. }\end{cases}
$$

In total, sum up all the $M_{\kappa}\left(\mathcal{F}_{i}, \mathcal{F}_{i}\right)$ for $1 \leq i, j \leq 3$ and the result follows from Theorem 1 and Theorem 2 ,

Remark. The case $d^{\prime}=d=1$ has been shown in [31], Theorem 23 and 25 . 


\section{Conclusion}

In this paper we have studied the exponential sums $\sum_{x \in \mathbb{F}_{q}}(-1)^{\operatorname{Tr}_{1}^{m}\left(\alpha x^{2^{m}+1}\right)+\operatorname{Tr}_{1}^{n}\left(\beta x^{2^{k}+1}\right)}$ and $\sum_{x \in \mathbb{F}_{q}}(-1)^{\operatorname{Tr}_{1}^{m}\left(\alpha x^{2^{m}+1}\right)+\operatorname{Tr}_{1}^{n}\left(\beta x^{2^{k}+1}+\gamma x\right)}$ with $\alpha \in \mathbb{F}_{2^{m}},(\beta, \gamma) \in \mathbb{F}_{q}^{2}$. After giving the value distribution of $\sum_{x \in \mathbb{F}_{q}} \zeta_{p}^{\operatorname{Tr}_{1}^{m}\left(\alpha x^{2^{m}+1}\right)+\operatorname{Tr}_{1}^{n}\left(\beta x^{2^{k}+1}\right)}$ and $\sum_{x \in \mathbb{F}_{q}}(-1)^{\operatorname{Tr}_{1}^{m}\left(\alpha x^{2^{m}+1}\right)+\operatorname{Tr}_{1}^{n}\left(\beta x^{2^{k}+1}+\gamma x\right)}$, we determine the correlation distribution among a family of sequences, and the weight distributions of the cyclic $\operatorname{codes} \mathcal{C}_{1}$ and $\mathcal{C}_{2}$. These results generalize [17], [27] and [31].

\section{Acknowledgements}

The authors will thank the anonymous referees for their helpful comments.

\section{References}

[1] A.W. Bluher, "On $x^{q+1}+a x+b$," Finite Fields and Their Appli., vol. 10, pp. 285-305, 2004.

[2] A. Canteaut, P. Charpin, and H. Dobbertin, "Binary m-sequences with three-valued cross-correlation: a proof of Welchs conjecture," IEEE Trans. Inform. Theory, vol. 46, no. 1, pp. 4-8, Jan. 2000.

[3] R.S. Coulter, "ON the evaluation of a class of Weil sums in characteristic 2," New Zeal. Jour. Math., vol. 28, pp. 171-184, 1999.

[4] T. Cusick and H. Dobbertin, "Some new three-valued cross-correlation functions for binary m-sequences," IEEE Trans. Inform. Theory, vol. 42, no. 4, pp. 1238-1240, April 1996.

[5] C. Ding, T. Helleseth, and K.Y. Lam, "Several classes of binary sequences with three-level autocorrelation," IEEE Trans. Inf. Theory, vol. 45, no. 7, pp. 2606-2612, Nov. 1999.

[6] C. Ding, T. Helleseth, and H. Martinsen, "New families of binary sequences with optimal three-valued autocorrelation," IEEE Trans. Inf. Theory, vol. 47, no. 1, pp. 428-433, Jan. 2001. 
[7] H. Dobbertin, P. Felke, T. Helleseth, and P. Rosendahl, "Niho type crosscorrelation functions via Dickson polynomials and Kloosterman sums," IEEE Trans. Inform. Theory, vol. 52, no. 2, pp. 613-627, Feb. 2006.

[8] R. Gold. "Maximal recursive sequences with 3-valued recursive crosscorrelation functions," IEEE Trans. Inform. Theory, vol. 14, no. 1, pp. 154-156, Jan.1968.

[9] S.W. Golomb and G. Gong, Signal Design for Good Correlation-For Wireless Communication, Cryptography and Radar. New York: Cambridge Univ. Press, 2005.

[10] T. Helleseth, "Some results about the cross-correlation function between two maximal linear sequences," Discrete Math., vol. 16, no. 3, pp. 209232,1976 .

[11] T. Helleseth, "A note on the cross-correlation function between two binary maximal length linear sequences," Discrete Math., vol. 23, no. 3, pp. 301307, 1978.

[12] T. Helleseth, A. Kholosha, and G.J. Ness, "Characterization of m-Sequences of lengths $2^{2 k}-1$ and $2^{k}-1$ with three-valued cross correlation," IEEE Trans. Inform. Theory, vol. 53, no. 6, pp. 2236-2245, June 2007.

[13] T. Helleseth and P.V. Kumar, "Sequences with low correlation," in Handbook of Coding Theory, V. S. Pless and W. C. Huffman, Eds. Amsterdam, The Netherlands: North-Holland, 1998.

[14] H. Hollmann and Q. Xiang, "A proof of Welch and Niho conjectures on cross-correlation of binary m-sequences," Finite Fields and Their Appli., vol. 7, no. 2, pp. 253-286, 2001.

[15] , L. Hu, X. Zeng, N. LI, and W. Jiang, Period-different m-sequences with at most a four-valued cross correlation, to appear.

[16] J. Luo, Y. Tang and H. Wang "Exponential sums, cyclic codes and sequences: the odd characteristic Kasami case," to appear.

[17] T. Kasami, "Weight distribution of Bose-Chaudhuri-Hocquenghem codes," in Combinatorial Mathematics and Its Applications. R. C. Bose and T. A. Dowling, Eds. Chapel Hill, NC: Univerisity of North Carolina Press, 1969, pp. 335-357. 
[18] T. Kasami, "Weight distribution formula for some class of cyclic codes," Coordinated Sci. Lab., Univ. Illinois, Urabana-Champaign, Tech. Rep. R285(AD 635274), 1966.

[19] T. Kasami, "Weight enumerators for several classes of subcodes of the second order binary Reed-Muller codes," Inf. and Control, vol. 18, pp. 369-394, 1971 .

[20] R. Lidl, and H. Niederreiter, Finite Fields, Addison-Wesley, Encyclopedia of Mathematics and its Applications, vol. 20, 1983.

[21] J. Lahtonen, "Two remarks on a paper by Moreno and Kumar," IEEE Trans. Inf. Theory, vol. 41, no. 3, pp. 859-861, May 1995.

[22] O. Moreno and P.V. Kumar, "Minimum distance bounds for cyclic codes and Deligne's Theorem," IEEE Trans. Inf. Theory, vol. 39, no. 5, pp. 15241534, Sept. 1993.

[23] G. J. Ness and T. Helleseth, "A new family of four-valued cross correlation between m-sequences of different lengths," IEEE Trans. Inf. Theory, vol. 53, no. 11, pp. 4308-4313, Nov. 2007.

[24] Y. Niho, "Multivalued cross-correlation functions between two maximal linear recursive sequences," Ph.D dissertation, Univ. South.Calif., Los Angles, 1972 .

[24] K.G. Paterson "Applications of exponential sums in communications theory," Extended Enterprise Laboratory, HP Laboratories Bristol, HPL-1999101, 13th Sept., 1999.

[25] P. Rosendahl, "Niho type cross-correlation functions and related equations," Ph.D. dissertation, Department of Computer Science, Univ. Turku, Turku, Finland 2004.

[26] M.K. Simon, J. Omura, R. Scholtz, and K. Levitt, "Spread Spectrum Communications," Rockville, MD: Computer Science, 1985, vol.ICIII.

[27] M. Van Der Vlugt, "Surfaces and the weight distribution of a family of codes," IEEE Trans. Inf. Theory, vol. 43, no. 4, pp. 1354-1360, Apri. 1997. 
[28] N.Y. Yu and G. Gong, "A new binary sequence family with low correlation and large size,", IEEE Trans. Inf. Theory, vol. 52, no. 4, pp. 1624-1636, April 2006.

[29] N.Y. Yu and G. Gong, "New binary sequences with optimal autocorrelation magnitude," IEEE Trans. Inf. Theory, vol. 54, no. 10, pp. 4771-4779, Oct. 2008 .

[30] X. Zeng, N. Li and L. Hu, "A class of nonbinary codes and their weight distribution," see http://arxiv.org/abs/0802.3430, preprint.

[31] X. Zeng, J.Q. Liu and L. Hu, "Generalized Kasami sequences: the large set," IEEE Trans. Inf. Theory, vol. 53, no. 7, pp. 2578-2598, July 2007. 